\title{
ЭКСПЕРИМЕНТАЛЬНАЯ ДИНАМИКА
}

\section{О финальном движении \\ скользящих и вращающихся дисков \\ с однородным кулоновым трением}

\author{
П. Д. Вайдман, Ч. Мальотра
}

От редакции. Продолжая публикации в этом новом разделе, призванном дополнить аналитические исследования в динамике экспериментом, мы приводим перевод данной работы, по своему содержанию примыкающей к работе Фаркаша и др., опубикованной в предыдущем номере (НД, т. 7, № 1, с. 139-146).

Мы провели обзор проделанных ранее исследований, касающихся финального движения дисков по горизонтальной плоскости с однородным сухим трением. Во всех предыдущих исследованиях было показано, что для тонкого круглого кольца или для однородного круглого диска радиуса $R$ скольжение и вращение прекращаются одновременно. Более того, при произвольных ненулевых начальных значениях скорости скольжения диска $v$ и угловой скорости вращения $\omega$ предельное значение отношения скоростей $\epsilon_{0}=v / R \omega$ всегда равно 1.0 для кольца и 0.653 для однородного диска. В данной работе нами показано, что для кольцевого диска с отношением внешнего и внутреннего радиусов $\eta=R_{2} / R_{1}$ скольжение и вращение прекращаются одновременно, однако значение выражения $\epsilon_{0}$ будет зависеть от $\eta$. Если рассмотреть диск, составленный из двух концентрических дисков, причем нижний диск имеет радиус $R_{1}$ и толщину $H_{1}$, а верхний - толщину $H_{2}$ и радиус $R_{2}$, то для такого составного диска выражение $\epsilon_{0}$ будет зависеть не только от $\eta$, но и от отношения $\lambda=H_{1} / H_{2}$. Скольжение и вращение будут прекращаться одновременно, но величина $\epsilon_{0}$ будет стремиться к нулю при $k>\sqrt{2 / 3}$, будет постоянной ненулевой величиной при $1 / 2<k<\sqrt{2 / 3}$ и будет стремиться к бесконечности при $k<1 / 2$. Здесь $k-$ безразмерный радиус инерции составного диска, т. е. его радиус инерции, разделенный на радиус того диска, который находится в контакте с плоскостью. Эти выводы о трех режимах движения согласуются с выводами, полученными в статье [2] геометрическими методами для обобщенных осесимметричных тел с изменяющимся радиусом инерции. Новые эксперименты с ПВХ-дисками,

Weidman P.D., Malhotra Ch. P. On the terminal motion of sliding spinning disks with uniform Coulomb friction // Physica D: Nonlinear Phenomena, 2007, vol. 233, no. 1, pp. 1-13. (C) 2007 Elsevier. Перевод с английского А. С. Кулешова, под редакцией А. П. Иванова.

Патрик Д. Вайдман

Department of Mechanical Engineering, University of Colorado, Boulder, CO 80309-0427, USA

Читан Мальотра

Tata Research Development and Design Centre, 54/B Hadapsar Industrial Estate, Pune 411013, India

НЕЛИНЕЙНАЯ ДИНАМИКА 2011. Т. 7. №2. С. 339-365 
скользящими по нейлоновой ткани, натянутой на горизонтальную поверхность из плексигласа лишь частично подтверждают наличие трех указанных режимов движения, т. е. на основании проведенных экспериментов был сделан вывод о необходимости привлечения более сложных моделей трения для описания движения диска по плоскости.

Ключевые слова: динамика твердого тела, финальное движение, нелинейное поведение

\section{1. Введение}

Задача о движении скользящих и вращающихся тел имеет довольно длительную историю, восходящую, по меньшей мере, к работам Н. Е. Жуковского [1]. Обзор работ, опубликованных по данной теме до 1991 года, приведен в статьях [2, 3], где было отмечено, что многие результаты переоткрывались разными авторами по два, а то и по три раза, вследствие того, что эти авторы не были знакомы с работами предшественников.

Мы начнем с того, что внесем некоторые уточнения в историю вопроса. Жуковский [1], рассматривая однородное трение в каждой точке контакта, ввел функцию момента, которая полностью характеризует связь между силой трения скольжения и моментом трения. Функция момента $M(x, y)$ - это результирующий момент трения относительно точки с координатами $(x, y)$, если тело приведено во вращение относительно этой точки. Хотя работа Жуковского применима к описанию любого движения со скольжением, его интерес заключался в том, чтобы дать описание зарождения и начала движения: какая комбинация силы и момента заставляет тело начать скольжение?

В противоположность вопросу о зарождении движения, можно рассмотреть задачу о финальном движении твердых тел, скользящих и вращающихся на плоскости. Возможно, наиболее простой задачей в этом случае является задача о предельном поведении (т. е. поведении непосредственно перед остановкой) тонкого кольца или однородного круглого диска, скользящих по плоской горизонтальной поверхности с однородным сухим трением. Обозначим $R$ - радиус диска или кольца, $v-$ линейную скорость центра и $\omega-$ угловую скорость. Первое наиболее полное аналитическое исследование данной задачи было проведено в работе А. Ю.Ишлинского с соавторами [4]. Для кольца ими было доказано, что при ненулевых начальных условиях для $v$ и $\omega$ скольжение и вращение прекращаются одновременно, а предельное поведение кольца описывается формулой $v=R \omega$. Они также установили, что для однородного диска скольжение и верчение прекращаются одновременно, а предельное поведение диска описывается формулой $v=k R \omega$, где значение $k$ (подсчитанное ими неверно) равно $k=0.71$. Это было поразительное открытие; для кольца и диска отношение линейной скорости центра к скорости точки на ободе стремится к некоторой фиксированной величине независимо от ненулевых начальных воздействий, приводящих объект в движение.

Независимо от работы советских специалистов, К. Военли и Э. Эриксен [5] также изучали предельное движение кольца и диска, скользящих по горизонтальной плоскости с однородным трением. Они первыми доказали, что для плитки с осесимметричным относительно центра масс распределением массы центр масс будет двигаться прямолинейно при одновременном наличии скольжения и качения. Они также показали, что в случае кольца предельное движение определяется соотношением $v=R \omega$, что соответствует результату, полученному в работе [4]. Для однородного диска ими было установлено, что скольжение и вращение прекращаются одновременно, а предельное поведение диска описывается формулой $v=0.65 R \omega$. Сами того не зная, Военли и Эриксен [5] подправили результат 
А. Ю. Ишлинского с соавторами [4], получив правильное значение $k^{*}=0.65$ вместо неправильного $k^{*}=0.71$, полученного в работе [4].

Гойял с соавторами $[2,3]$ провели обзор упомянутых выше результатов и обобщили их несколькими способами. В работе [2] авторы предложили два независимых геометрических описания для результирующей силы трения и момента трения между твердым телом и плоской поверхностью, по которой оно скользит. Одно из описаний использует функцию момента, предложенную Жуковским [1], тогда как в другом используется концепция предельных поверхностей, взятая из классической теории пластичности. Предельная поверхность - это граница множества всевозможных наборов силы и момента трения в пространстве сил и моментов. Предельные поверхности могут применяться при описании моделей с анизотропным трением, например, при описании скольжения коньков, тогда как функция момента применяется лишь для однородного трения. Подробный обзор концепции предельных поверхностей приведен в книге Мейсона [6]. Приложение теории предельных поверхностей к описанию движения твердого тела было проведено в [3], при этом так же, как и в [4, 5], были рассмотрены случаи кольца и однородного диска. Для кольца было установлено то же поведение, что и описанное в $[4,5]$, для диска была получена более точная формула, связывающая $v$ и $\omega R: v=0.653 \omega R$.

Наибольший интерес для нашей работы представляют результаты, полученные в [3] для тела с изменяющимся моментом инерции. Представим себе невесомое кольцо, к которому сверху приклеена невесомая доска, а масса распределена на доске по окружности радиуса $k$, концентрической с кольцом. В этом случае в зависимости от выбранного радиуса $k$ меняется выражение $R$, равное отношению радиуса кольца к радиусу инерции системы. Воспользуемся на время этими обозначениями, чтобы сформулировать в терминах $R$ полученные в [3] результаты:

1. При $R>\sqrt{2}$ все начальные движения (за исключением чистого вращения) свободно скользящего кольца в пределе стремятся к поступательному движению.

2. Для $R<1 / \sqrt{2}$ все начальные движения (за исключением поступательного движения) кольца в пределе стремятся к вращению вокруг центра кольца (Гойял в своей работе допустил ошибку, написав $R>1 / \sqrt{2}$ ).

3. При $1 / \sqrt{2} \leqslant R \leqslant \sqrt{2}$ финальное движение зависит от $R$ и изменяется от чистого вращения до поступательного движения.

Здесь под «финальным движением» понимается асимптотическое отношение величины линейной скорости центра масс $v$ к выражению $\omega R$ непосредственно перед полной остановкой. Например, в случае 1, описанном выше, это отношение стремится к бесконечности, даже несмотря на то, что линейная скорость тела убывает. Но обратиться в нуль она не может, так как это должно произойти одновременно с прекращением вращения.

В статье Гойяла с соавторами приводится также ссылка на работу [7], в которой для диска с равномерным давлением на опорную плоскость (в отличие от кольца) также имеется три случая финальных движений, описываемых условиями вида 1-3. Надо только везде заменить $1 / \sqrt{2}$ на $\sqrt{3 / 2}$, а $\sqrt{2}$ - на 2 соответственно. Свои результаты авторы распространили также на произвольное распределение массы и сил трения, рассмотрев случаи анизотропного трения и неоднородных контактных напряжений. Они показали, что в типичном случае для финального движения отношение $v$ к $\omega R$ лежит в некоторых границах, определяемых только инерционными свойствами тела и распределением сил трения. Граничные значения соответствуют точкам, в которых радиус предельной поверхности параллелен нормали к ней. Гойял с соавторами обсуждали также особые ситуации, когда предельное движение 
будет чистым вращением или поступательным движением в течение конечного промежутка времени. Тот же вопрос будет обсуждаться ниже в связи с новыми экспериментальными результатами, полученными в данной работе.

Недавно Фаркаш с соавторами [8] независимо от предшествующих исследований данной задачи установил, что для однородного диска, стартовавшего по плоскости с ненулевой скоростью центра масс и ненулевой угловой скоростью, предельное соотношение имеет вид $\epsilon_{0}=v / \omega R=0.653$. Результаты работы Фаркаша [8], упомянутые также в работе Хелси [9], опубликованной в журнале Nature, уже в третий раз повторяют основной вывод. Мотивом исследований Фаркаша и его соавторов [8] был вопрос о том, насколько плотно друг к другу примыкают частицы пыли, имеющие форму правильных многогранников $[10,12]$. Проведенные в [8] исследования чисто аналитические, с использованием полных эллиптических интегралов первого и второго рода. Как и предыдущие исследования, работа Фаркаша основывалась на предположении о равномерности нормального распределения давления диска на плоскость и на том, что локально, для каждой точки диска, находящейся в контакте с плоскостью, сила трения не зависит от скорости и пропорциональна силе нормального давления. Если обозначить через $H$ толщину диска, то распределение давлений будет близко к однородному, когда отношение $H / R$ достаточно мало. Как отмечено выше, количественные и качественные результаты о динамике поведения дисков на плоскости, описанные Фаркашем с соавторами [8], уже были получены до этого в более общей форме Гойялом с соавторами $[2,3]$, рассматривавшем объекты более сложной формы, чем однородный диск. Например, используя свойство моментной функции Жуковского $\left(F_{x}, F_{y}\right)=$ $=(\partial M / \partial x,-\partial M \partial y)$, легко можно получить один из другого графики для силы и момента трения, приведенные на рисунке 2а статьи [8]. То же относится и к рисунку 2b.

Наш интерес к данной проблеме возник вследствие явлений, упомянутых авторами работы [8] и замеченных ими в кафе или пивных барах. Монеты и пробки от пивных бутылок, действительно, вроде бы прекращают свое скольжение и вращение одновременно, однако было трудно различить момент, после которого движение уже может рассматриваться как предельное. Имея похожий объект большего размера, мы запустили его по столу и нам показалось, что сначала он остановился, а затем прекратил вращение. Данный эффект не связан с продолговатой формой образца, но определяется тем, что образец имеет слегка выпуклую наружу поверхность и, следовательно, его контакт со столом происходил по малой площадке, радиус которой был существенно меньше, чем радиус инерции образца. И тогда мы начали исследование с тел, обладающих геометрией, отличной от диска, чтобы увидеть, в каких случаях финальное движение рассматриваемого объекта может отличаться от движения диска.

Примечание. Подобным образом Гойлл и его соавторы решили исследовать разные типы финальных движений после того, как в перерыве хоккейного матча они увидели, как мужчина, одетый в костюм медведя, прокатился на брюхе по лъду и в конце своего движения пришел в чистое вращение.

В работе [13] мы кратко сформулировали полученные нами результаты, распространяющие результат работы Фаркаша на случай двойного диска, для которого были найдены различные типы финальных движений. На момент, когда мы подавали рукопись статьи [13] в редакцию, нам не была знакома работа Гойяла и ссылки на литературу, содержащиеся в ней: об этой работе мы узнали от рецензентов нашей статьи [13]. Таким образом, цель настоящей статьи является троякой. Прежде всего мы расскажем о новых результатах относительно финальных движений кольцевого диска, такого, как изображен на рисунке 1а. 
Он был упомянут Гойялом и его соавторами, но его поведение детально не исследовалось. Во-вторых, мы представим полный отчет о результатах, полученных для двойного составного диска, изображенного на рисунке $1 \mathrm{~b}$, включая подробные вычисления, не сообщенные в [13]. И в-третьих, мы представим результаты экспериментов с составным диском, которые частично согласуются с наиболее простыми теоретическими выгодами.
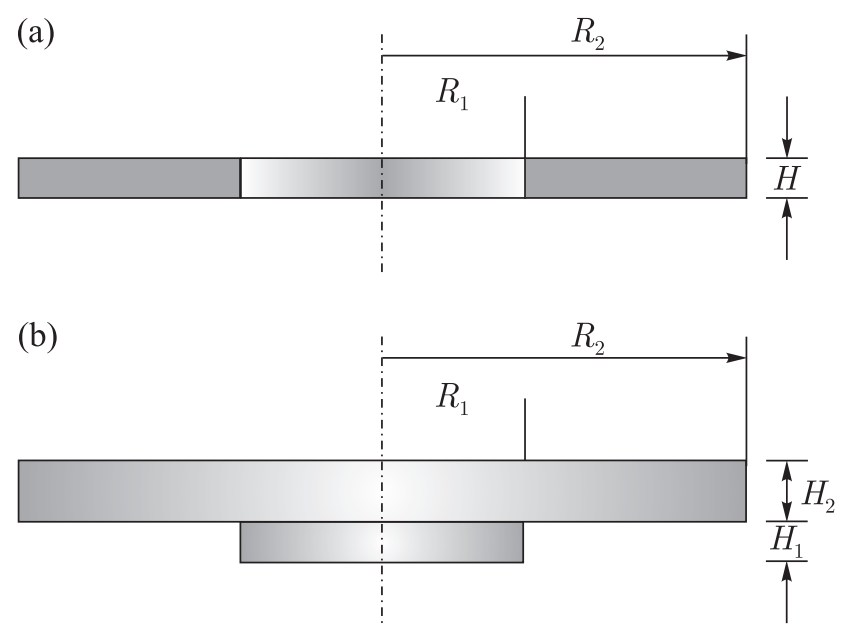

Рис. 1. Геометрия кольцевого диска (а) и составного двойного диска (b).

Статья построена следующим образом. Во второй части мы, следуя работе Фаркаша и его соавторов, получаем некоторые теоретические результаты, применимые к осесимметричных телам, отличным от диска. Попутно мы исправляем типографские опечатки, допущенные в статье [8]. В части 3 предельное движение кольцевого диска определяется как функция введенного выше параметра $\eta$. В части 4 предельное движение сложного составного диска анализируется в терминах нормализованного радиуса инерции $k$. Экспериментальные результаты, полученные путем анализа видео скользящего и вращающегося диска, изложены в части 5 , а часть 6 содержит заключительные выводы и замечания.

\section{2. Элементы теории}

При вычислении силы и момента трения, действующих на произвольный осесимметричный диск, совершающий плоскопараллельное движение по шероховатой плоскости с однородным сухим трением, мы будем пользоваться обозначениями работы [8]. Закон трения Кулона утверждает, что величина локальной силы трения пропорциональна локальной силе нормального давления, а направление локальной силы трения противоположно направлению относительной скорости той точки, в которой происходит контакт. Для равномерного распределения нормального давления сила трения, действующая на диск, определяется формулой

$$
\mathbf{F}=-\frac{\mu F_{n}}{A} \int_{A} \frac{\mathbf{v}+\boldsymbol{\omega} \times \mathbf{r}}{|\mathbf{v}+\boldsymbol{\omega} \times \mathbf{r}|} d S,
$$

где $\mathbf{r}-$ радиус-вектор из центра диска, $\mathbf{v}-$ линейная скорость центра диска, $\boldsymbol{\omega}-$ угловая скорость диска, $\mu$ - постоянный коэффициент трения, $A-$ площадь части диска, находящейся в контакте с горизонтальной поверхностью, и $F_{n}=m g-$ нормальная реакция, 
действующая на диск массы $m$, движущийся в поле силы тяжести $g$. Аналогично, момент силы трения определяется формулой

$$
\mathbf{T}=-\frac{\mu F_{n}}{A} \int_{A} \mathbf{r} \times \frac{\mathbf{v}+\boldsymbol{\omega} \times \mathbf{r}}{|\mathbf{v}+\boldsymbol{\omega} \times \mathbf{r}|} d S .
$$

Вычисление выражений (1) и (2), выполненное в работе Фаркаша с соавторами [8] для однородного диска радиуса $R$, дает: $\mathbf{F}=-F_{n} \mathcal{F}(\epsilon) \mathbf{e}_{v}$ и $\mathbf{T}=-F_{n} R \mathcal{T}(\epsilon) \mathbf{e}_{\omega}$, где $\mathbf{e}_{v}=$ $=\mathbf{v} / v-$ единичный вектор вдоль направления поступательного перемещения, $\mathbf{e}_{\omega}=\boldsymbol{\omega} / \omega-$ единичный вектор, нормальный к плоскости диска, а $\mathcal{F}(\epsilon), \mathcal{T}(\epsilon)$ выражаются при помощи полных эллиптических интегралов $K(\epsilon)$ и $E(\epsilon)$. Приведенные в работе [8] формулы для $\mathcal{F}(\epsilon)$ и $\mathcal{T}(\epsilon)$ - неправильные, хотя графики соответствующих функций, изображенные на рисунке 2 работы [8], являются верными. В соответствии с обозначениями книги [14] имеем:

$$
\mathcal{F}(\epsilon)=\left\{\begin{array}{l}
\frac{4}{3 \pi \epsilon}\left[\left(\epsilon^{2}+1\right) E\left(\epsilon^{2}\right)+\left(\epsilon^{2}-1\right) K\left(\epsilon^{2}\right)\right], \quad \epsilon \leqslant 1, \\
\frac{4}{3 \pi}\left[\left(\epsilon^{2}+1\right) E\left(\epsilon^{-2}\right)+\left(\epsilon^{2}-1\right) K\left(\epsilon^{-2}\right)\right], \quad \epsilon \geqslant 1,
\end{array}\right.
$$

и

$$
\mathcal{T}(\epsilon)=\left\{\begin{array}{l}
\frac{4}{9 \pi}\left[\left(4-2 \epsilon^{2}\right) E\left(\epsilon^{2}\right)+\left(\epsilon^{2}-1\right) K\left(\epsilon^{2}\right)\right], \quad \epsilon \leqslant 1, \\
\frac{4 \epsilon}{9 \pi}\left[\left(4-2 \epsilon^{2}\right) E\left(\epsilon^{-2}\right)+\left(2 \epsilon^{2}-5+3 \epsilon^{-2}\right) K\left(\epsilon^{-2}\right)\right], \quad \epsilon \geqslant 1 .
\end{array}\right.
$$

Для громоздких вычислений, использующих программу Mathematica [15], полезно иметь выражения для $\mathcal{F}(\epsilon)$ и $\mathcal{T}(\epsilon)$, справедливые при всех $\epsilon$. Эти результаты, сообщенные нам Мадьяри и Томасом (частное сообщение) представлены ниже в терминах неполных эллиптических интегралов $E(\varphi, \alpha)$ и $F(\varphi, \alpha)$ в соответствии с определениями из [14]:

$$
\begin{aligned}
\mathcal{F}(\epsilon) & =\frac{1+\epsilon}{3 \pi \epsilon}\left\{\left(1+\epsilon^{2}\right)\left[E\left(\frac{\pi}{4}, \alpha\right)+E\left(\frac{3 \pi}{4}, \alpha\right)\right]-\right. \\
& \left.-(1-\epsilon)^{2}\left[F\left(\frac{\pi}{4}, \alpha\right)+F\left(\frac{3 \pi}{4}, \alpha\right)\right]\right\}, \\
\mathcal{T}(\epsilon)= & \frac{2(1+\epsilon)}{9 \pi}\left\{\left(2-\epsilon^{2}\right)\left[E\left(\frac{\pi}{4}, \alpha\right)+E\left(\frac{3 \pi}{4}, \alpha\right)\right]+\right. \\
+ & \left.(1-\epsilon)^{2}\left[F\left(\frac{\pi}{4}, \alpha\right)+F\left(\frac{3 \pi}{4}, \alpha\right)\right]\right\},
\end{aligned}
$$

где $\alpha=4 \epsilon /(1+\epsilon)^{2}$.

Таким образом, движение диска описывается двумя скалярными уравнениями, следующими из закона изменения импульса и закона изменения кинетического момента диска:

$$
\begin{gathered}
F=m \frac{d v}{d t}, \\
T=I \frac{d \omega}{d t},
\end{gathered}
$$

где $I$ - осевой момент инерции диска. Эти два уравнения могут быть приведены к одному уравнению, неподвижная точка которого соответствует предельному движению диска. Для однородного сплошного диска Фаркаш с соавторами переоткрыли неподвижную точку $\epsilon_{0}=0.653$ и сделали, таким образом, вывод, что скольжение и вращение диска прекращаются одновременно, независимо от начальных условий. 


\section{3. Кольцевой диск}

Рассмотрим сначала движение кольцевого диска, изображенного на рисунке $1 \mathrm{a}$, с толщиной $H$, внутренним радиусом $R_{1}$, внешним радиусом $R_{2}$ и введем $\eta=R_{1} / R_{2}$ - отношение радиусов. При $H / R_{2} \ll 1$ распределение нормального давления при движении диска близко к равномерному и равно $m g / \pi\left(R_{2}^{2}-R_{1}^{2}\right)$. При этих предположениях уравнения (1) и (2) справедливы, и мы получаем:

$$
\begin{aligned}
& \mathbf{F}=-\mu m g\left[\frac{1}{1-\eta^{2}} \mathcal{F}(\epsilon)-\frac{\eta^{2}}{1-\eta^{2}} \mathcal{F}(\epsilon / \eta)\right] \mathbf{e}_{v}, \\
& \mathbf{T}=-\mu m g\left[\frac{R_{2}}{1-\eta^{2}} \mathcal{T}(\epsilon)-\frac{R_{1} \eta^{2}}{1-\eta^{2}} \mathcal{T}(\epsilon / \eta)\right] \mathbf{e}_{\omega},
\end{aligned}
$$

где $\epsilon=v / \omega R_{2}$ - отношение скоростей в произвольный момент времени $t$. Масса и осевой момент инерции кольцевого диска равны

$$
m=\pi \rho H\left(R_{2}^{2}-R_{1}^{2}\right), \quad I=\frac{\pi \rho}{2} H\left(R_{2}^{4}-R_{1}^{4}\right),
$$

где $\rho$ - плотность, которую будем считать постоянной. Используя эти результаты, запишем уравнения $(5 a),(5 b)$ в виде

$$
\begin{gathered}
\frac{d v}{d t}=-\frac{\mu g}{\left(1-\eta^{2}\right)}\left[\mathcal{F}(\epsilon)-\eta^{2} \mathcal{F}(\epsilon / \eta)\right] \\
\frac{d \omega}{d t}=-\frac{2 \mu g}{R_{2}\left(1-\eta^{4}\right)}\left[\mathcal{T}(\epsilon)-\eta^{3} \mathcal{T}(\epsilon / \eta)\right] .
\end{gathered}
$$

Введем безразмерные переменные $v^{*}, \omega^{*}$ и безразмерное время $t^{*}$ по формулам

$$
v=v^{*} \mu \sqrt{R_{2} g}, \quad \omega=\omega^{*} \mu \sqrt{\frac{g}{R_{2}}}, \quad t=t^{*} \sqrt{\frac{R_{2}}{g}},
$$

тогда уравнения $(7 a)$ и $(7 b)$ можно переписать следующим образом:

$$
\begin{aligned}
\frac{d v^{*}}{d t^{*}} & =-\frac{1}{\left(1-\eta^{2}\right)}\left[\mathcal{F}(\epsilon)-\eta^{2} \mathcal{F}(\epsilon / \eta)\right], \\
\frac{d \omega^{*}}{d t^{*}} & =-\frac{2}{\left(1-\eta^{4}\right)}\left[\mathcal{T}(\epsilon)-\eta^{3} \mathcal{T}(\epsilon / \eta)\right] .
\end{aligned}
$$

Разделим теперь $(8 a)$ на $(8 b)$ и, следуя работе [8], введем вместо $\omega^{*}$ новую независимую переменную $x=-\ln \omega^{*}$. Тогда получим

$$
f(\epsilon, \eta) \equiv \frac{d \epsilon}{d x}=\epsilon-\frac{\left(1+\eta^{2}\right)}{2}\left[\frac{\mathcal{F}(\epsilon)-\eta^{2} \mathcal{F}(\epsilon / \eta)}{\mathcal{T}(\epsilon)-\eta^{3} \mathcal{T}(\epsilon / \eta)}\right] .
$$

Переменная $x$ играет важнейшую роль при анализе неподвижных точек динамической системы (9). Поскольку угловая скорость $\omega^{*}$ монотонно убывает со временем, то $x$ монотонно возрастает и, следовательно, может рассматриваться как «псевдовремя».

Если положить в этих формулах $\eta=0$, то мы получим уравнение (8) из работы [8], справедливое для сплошного диска. Так же, как и в случае сплошного диска, уравнение (9) для кольцевого диска имеет три неподвижные точки. Две из них $\epsilon=0$ и $\epsilon=\infty$ соответствуют 
чистому вращению и поступательному движению. Для всех других начальных условий, соответствующих комбинации вращения и скольжения, уравнение (9) имеет промежуточную притягивающую неподвижную точку $\epsilon_{0}$. Это видно из рисунка 2 , на котором изображен график функции $f(\epsilon, \eta)$, построенный с помощью программы Mathematica [15] при фиксированных значениях параметра $\eta: 0,0.25,0.50,0.75$ и 0.999. График зависимости неподвижной точки $\epsilon_{0}$ от $\eta$ позволяет утверждать, что $\epsilon_{0}$ меняется в пределах $0.653 \leqslant \epsilon_{0} \leqslant 1.0$. Величина $\epsilon_{0}=0.653$, получающаяся при $\eta=0$, соответствует случаю сплошного диска, а величина $\epsilon_{0}=1.0$, получающаяся при $\eta=1$, соответствует бесконечно тонкому кольцу высоты $H$. Так как предельное значение $\epsilon_{0}$ является конечным и ненулевым, то можно сделать вывод, что и для кольцевого диска скольжение и вращение прекращаются одновременно.

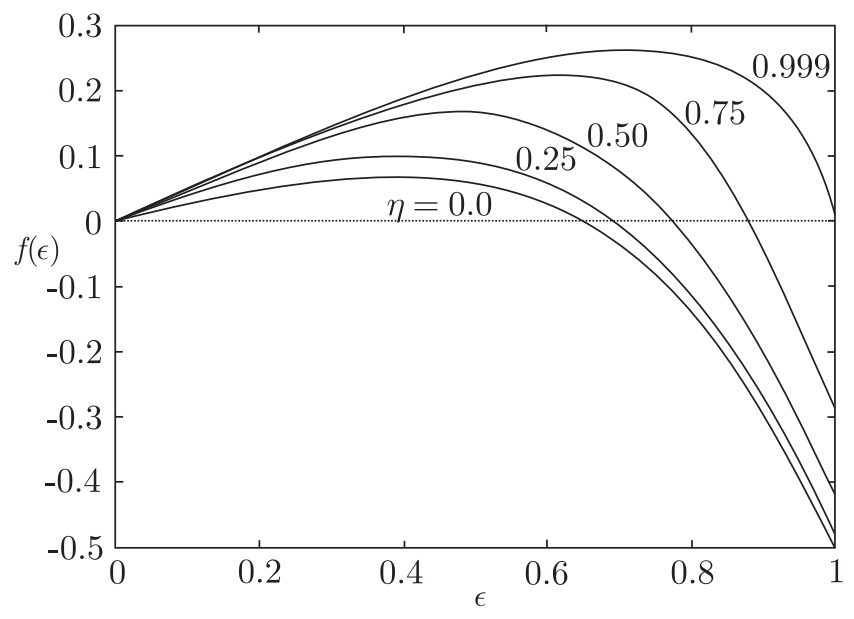

Рис. 2. Графики $f(\epsilon)$ для различных значений $\eta$ в случае кольцевого диска.

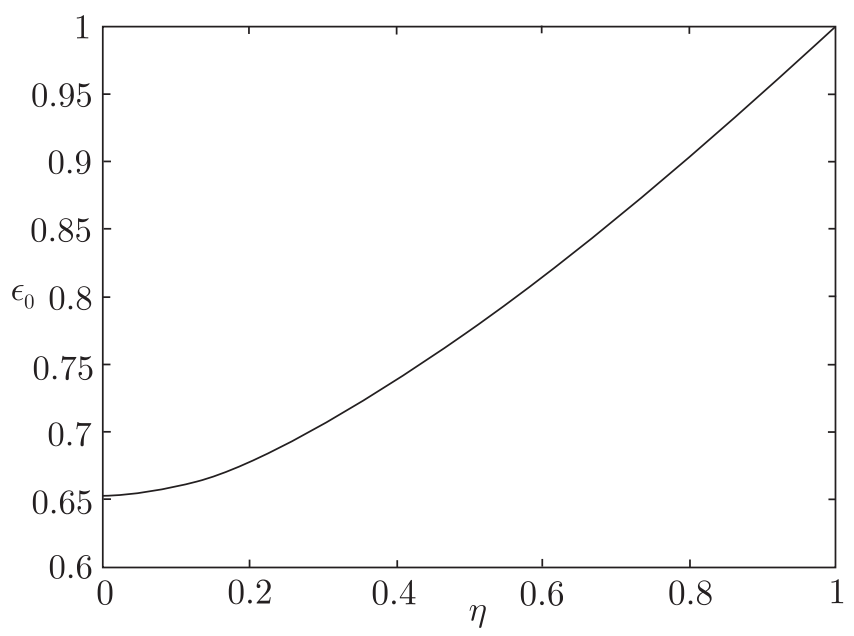

Рис. 3. Зависимость предельного отношения скоростей $\epsilon_{0}$ от параметра $\eta$.

На рисунке 4 показаны три примера движения кольцевого диска, каждый с одинаковыми начальными условиями $v^{*}=3.0$ и $\omega^{*}=2.0$, но с разными значениями $\eta$. Изменение безразмерной линейной скорости $v^{*}$ и угловой скорости $\omega^{*}$ в зависимости от безразмерного времени $t^{*}$ показано сплошной и пунктирной линиями соответственно. Рисунок 4а показывает зависимости $v^{*}$ и $\omega^{*}$ от $t^{*}$. Скольжение и вращение в этом случае прекращаются одновременно в момент $t_{f}^{*}=3.565$. На рисунке $4 \mathrm{~b}$ показаны те же зависимости при $\eta=$ 
$=0.8$, при этом время до остановки слегка возрастает и становится равным $t_{f}^{*}=3.677$. На рисунке 4с представлены результаты для тонкого кольца $\eta=0.999$; время до остановки при этом еще более возрастает: $t_{f}^{*}=3.965$. Заметим, что в последнем примере $v^{*}$ и $\omega^{*}$ имеют одинаковое предельное поведение, что подтверждает выводы, полученные ранее в статьях А. Ю. Ишлинского с соавторами [4], Военли и Эриксена [5] и Гойяла с соавторами $[2,3]$ о предельном поведении тонкого кольца.
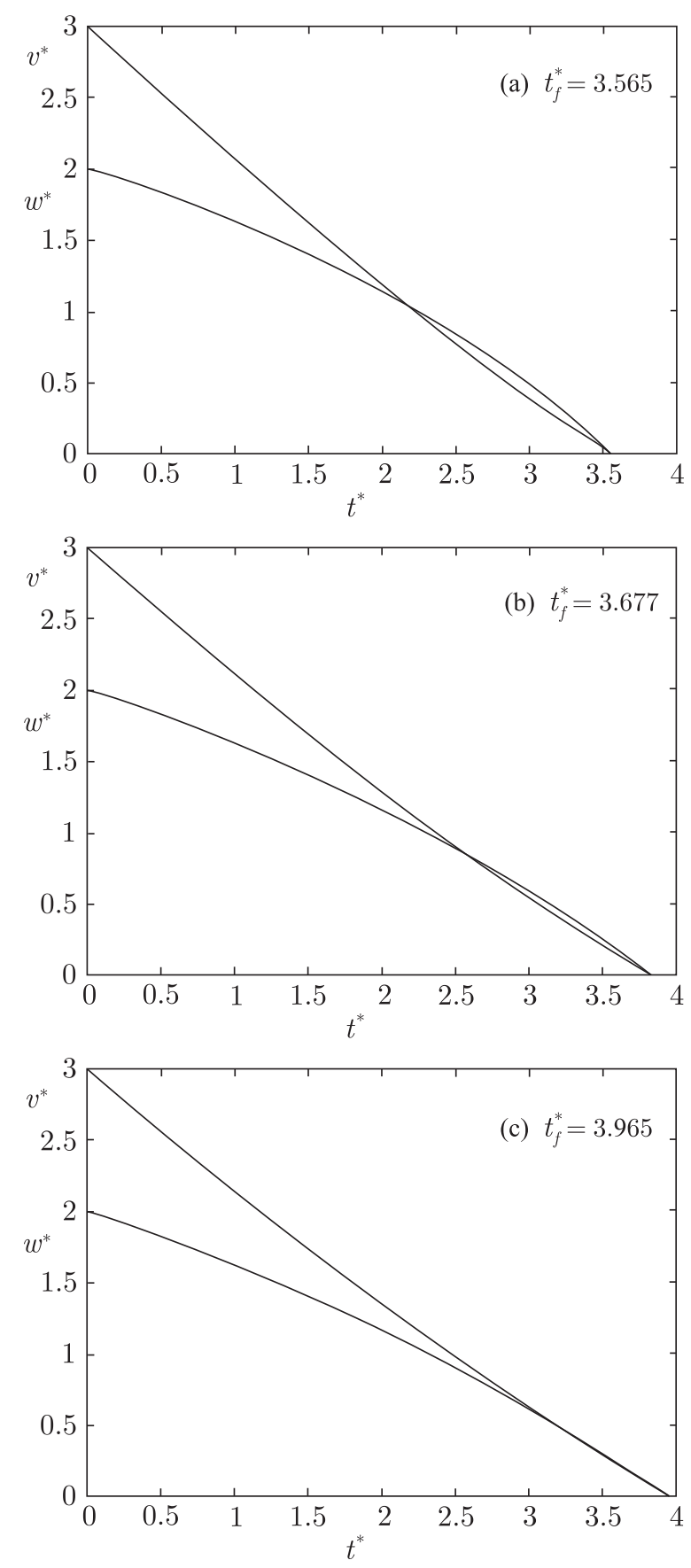

Рис. 4. Графики зависимости от времени линейной скорости (сплошная линия) и угловой скорости (пунктирная линия). Начальные условия: $v_{0}^{*}=3.0$ и $\omega_{0}^{*}=2.0$. а) Случай $\eta=0$ (сплошной диск); b) $\eta=0.8$; с) $\eta=0.999$ (тонкое кольцо). 


\section{4. Составной диск}

Эксперименты по движению выпуклой линзы по горизонтальному столу, в которых линза имеет большой осевой момент инерции, но малую площадку контакта со столом, побудили нас исследовать движение осесимметричного составного диска с однородной плотностью $\rho$. Как показано на рисунке $1 \mathrm{~b}$, нижний и верхний диски имеют радиусы $R_{1}$ и $R_{2}$ и толщины $H_{1}$ и $H_{2}$ соответственно. Такая конфигурация характеризуется двумя безразмерными параметрами: $\eta=R_{1} / R_{2}$ и $\lambda=H 1 / H 2$. Масса и осевой момент инерции составного диска равны, соответственно,

$$
m=\pi \rho\left(H_{1} R_{1}^{2}+H_{2} R_{2}^{2}\right), \quad I=\frac{\pi \rho}{2}\left(H_{1} R_{1}^{4}+H_{2} R_{2}^{4}\right) .
$$

Вычисление интегралов (1) и (2) для составного диска дает

$$
\begin{aligned}
& \mathbf{F}=-\mu m g \mathcal{F}(\epsilon) \mathbf{e})_{v}, \\
& \mathbf{T}=-\mu m g R_{1} \mathcal{T}(\epsilon) \mathbf{e}_{\omega},
\end{aligned}
$$

где $\epsilon=v / \omega R_{1}$ - отношение скоростей. Используя выражения (11a) и (11b), можно записать уравнения движения в виде

$$
\begin{gathered}
\frac{d v}{d t}=-\mu g \mathcal{F}(\epsilon), \\
\frac{d \omega}{d t}=-\frac{2 \mu g}{R_{2}}\left(\frac{\eta^{2} \lambda+1}{\eta^{4} \lambda+1}\right) \eta \mathcal{T}(\epsilon) .
\end{gathered}
$$

Вводя безразмерные переменные $v^{*}$ и $\omega^{*}$, а также безразмерное время $t^{*}$ по формулам

$$
v=v^{*} \mu \sqrt{R_{1} g}, \quad \omega=\omega^{*} \mu \sqrt{\frac{g}{R_{1}}}, \quad t=t * \sqrt{\frac{R_{1}}{g}},
$$

получаем уравнения в безразмерной форме:

$$
\begin{gathered}
\frac{d v^{*}}{d t^{*}}=-\mathcal{F}(\epsilon), \\
\frac{d \omega^{*}}{d t^{*}}=-\frac{2 \eta^{2}\left(\eta^{2} \lambda+1\right)}{\left(\eta^{4} \lambda+1\right)} \mathcal{T}(\epsilon) .
\end{gathered}
$$

Разделим $(13 a)$ на $(13 b)$ и сделаем замену независимой переменной по формуле $x=$ $=-\ln \omega^{*}$. В результате получим уравнение

$$
f(\epsilon, k) \equiv \frac{d \epsilon}{d x}=\epsilon-k^{2} \frac{\mathcal{F}(\epsilon)}{\mathcal{T}(\epsilon)} .
$$

Используя (10), мы находим, что $k$ в формуле (11) - это радиус инерции составного диска, поделенный на радиус диска, находящегося в контакте с плоскостью:

$$
k=\frac{1}{R_{1}} \sqrt{\frac{I}{m}}=\sqrt{\frac{\eta^{4} \lambda+1}{2 \eta^{2}\left(\eta^{2} \lambda+1\right)}} .
$$


Следовательно, семейство решений с двумя параметрами $\eta$ и $\lambda$ может быть проанализировано в терминах единственного параметра $k$. Заметим, что наш безразмерный радиус инерции $k$ является величиной, обратной величине $R$ из работы Гойяла с соавторами. На рисунке 5 показаны кривые $f(\epsilon)$ для некоторых фиксированных значений $k$. Можно заметить, что промежуток изменения $k-$ это $[0,+\infty]$. Кривая, для которой $k=k_{0}=1 / \sqrt{2}$, соответствует простому сплошному диску с притягивающей неподвижной точкой $\epsilon_{0}=0.653$, в которой график функции $f(\epsilon)$ пересекает горизонтальную ось. Мы исследуем финальное движение составного диска, увеличивая и уменьшая $k$ от значения $k_{0}$. При возрастании $k$ значение $\epsilon_{0}$ начинает убывать от величины $\epsilon_{0}=0.653$ и в конечном счете стремится к нулю, а график функции $f(\epsilon)$ меняется таким образом, что горизонтальная ось становится для него касательной; это происходит при $k_{1}=\sqrt{2 / 3}$ (см. рис. 5). При значениях $k$, превосходящих критическое значение $k_{1}$, получаются кривые, входящие в начало координат и не касающиеся горизонтальной оси, такие как $k_{2}$ и $k_{3}$. Таким образом, если $k>\sqrt{2 / 3}$, то единственной неподвижной точкой является значение $\epsilon_{0}=0$.

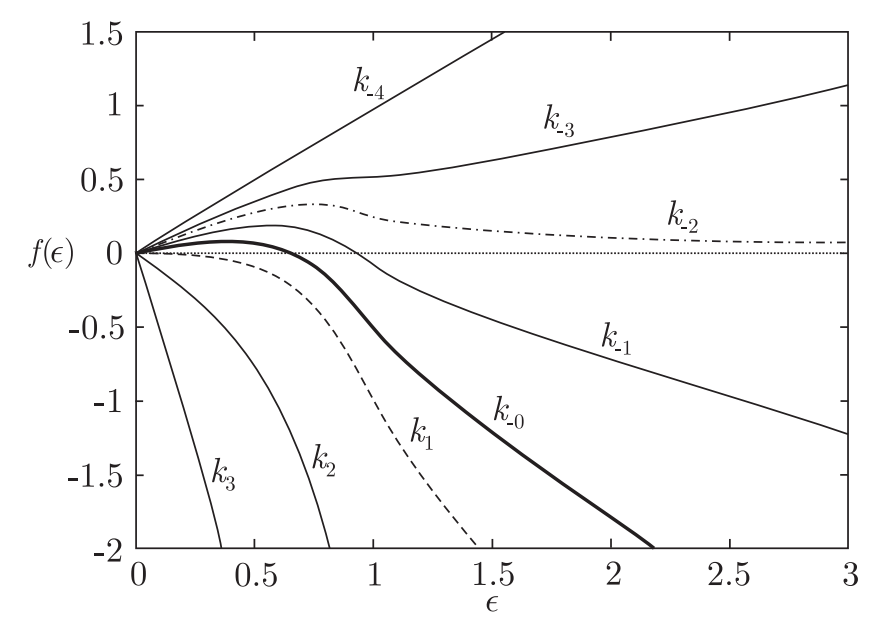

Рис. 5. Кривые $f(\epsilon)$ при различных значениях $k$ в случае составного диска. Для изображенных кривых: $k_{3}=2.0, k_{2}=1.2, k_{1}=\sqrt{2 / 3}, k_{0}=1 / \sqrt{2}, k_{-1}=0.60, k_{-2}=1 / 2, k_{-3}=0.40$ и $k_{-4}=0.10$. Пунктирная кривая $k_{1}$ касается горизонтальной оси в нуле; точечно-пунктирная кривая $k_{-2}$ касается горизонтальной оси в бесконечности; сплошная кривая $k_{0}$ соответствует случаю однородного диска.

Будем теперь уменьшать $k$ от значения $k_{0}$, соответствующего случаю однородного сплошного диска. При этом неподвижная точка функции $f(\epsilon)$ начнет смещаться вправо, что легко видеть из графика $k_{-1}$. При дальнейшем убывании $k$ неподвижная точка начинает стремиться к бесконечности, что показывает точечно-пунктирный график $k=k_{-2}=1 / 2$ на рисунке 5. В этом случае $f(\epsilon)$ касается оси $\epsilon$ в бесконечности. Кривые, соответствующие еще более малым значениям $k$, такие как $k=k_{-3}$ и $k=k_{-4}$, быстро поднимаются от горизонтальной оси, и угол наклона касательной к графику такой функции в начале координат возрастает. Таким образом, при всех значениях $k<1 / 2$ неподвижной точкой является точка $\epsilon_{0}=\infty$

Полученные выше прямым математическим анализом результаты, касающиеся движения составного диска, позволяют утверждать, что существует три предельных движения составного диска, информация о которых приведена в таблице 1. Эти режимы совпадают с режимами, полученными при помощи геометрических методов анализа в статьях Гойяла 
с соавторами $[2,3]$ для осесимметричного диска с радиально изменяющимся распределением масс. В приложении мы также воспользуемся данной методологией, чтобы получить три режима финального движения для кольца с переменным распределением масс, движение которого впервые было исследовано в работе Гойяла с соавторами и упоминалось во введении.

Таблица 1. Три режима предельного движения для составного диска, характеризуемого безразмерным радиусом инерции $k$

\begin{tabular}{cc}
\hline Режим & Предельное отношение скоростей \\
\hline$k<\frac{1}{2}$ & $\epsilon_{0}=0$ \\
$\frac{1}{2} k<\sqrt{\frac{2}{3}}$ & $0<\epsilon_{0}<\infty$ \\
$k>\sqrt{\frac{2}{3}}$ & $\epsilon_{0}=0$ \\
\hline
\end{tabular}

Полагая $f\left(\epsilon_{0}, k\right)=0$ в $(11)$, получим связь между радиусом инерции $k$ и предельной величиной $\epsilon_{0}$, а именно:

$$
k=\sqrt{\frac{\epsilon_{0} \eta \mathcal{T}\left(\epsilon_{0}\right)}{\eta \mathcal{F}\left(\epsilon_{0}\right)}} .
$$

График зависимости $k$ от $\epsilon_{0}$ представлен на рисунке 6 , причем жирная точка на графике соответствует случаю однородного сплошного диска.

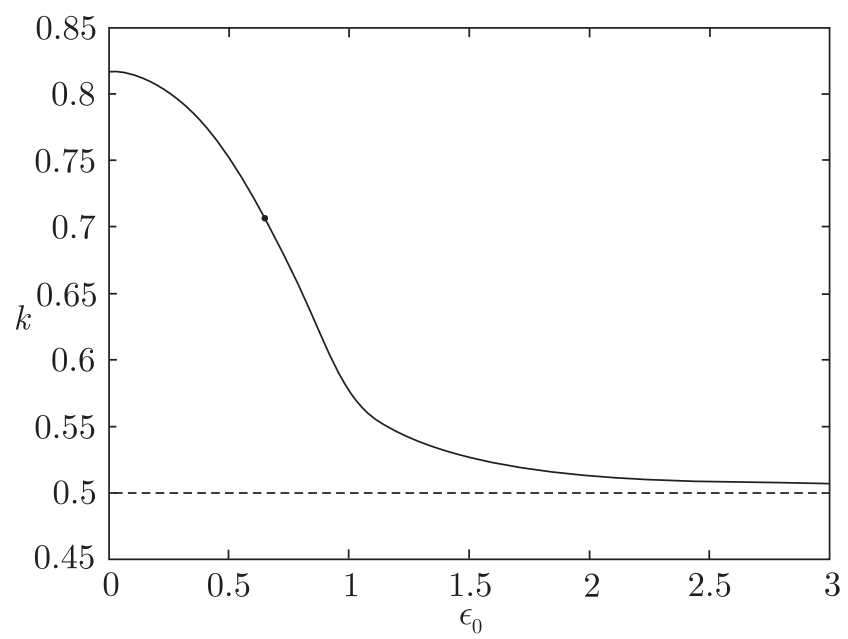

Рис. 6. График зависимости параметра $k$ от предельного отношения скоростей $\epsilon_{0}$. Точкой обозначен случай однородного сплошного диска $k=1 / \sqrt{2}, \epsilon_{0}=0.653$.

Рассмотрим теперь фундаментальное состояние - одно из тех, для которых $\epsilon_{0}$ является ненулевой константой. В конфигурационном пространстве $\eta-\lambda$ легко могут быть установлены границы перехода от этого режима к двум другим, перечисленным в таблице. Например, переход к предельному режиму движения $\epsilon_{0}=0$ может быть получен, если положить $k=\sqrt{2 / 3}$ в уравнении $(12)$, из которого затем находим

$$
\lambda \eta^{4}+4 \eta^{2}-3=0
$$


Таким образом, соответствующая граница определяется соотношением

$$
\lambda=\frac{3-4 \eta^{2}}{\eta^{4}} .
$$

Переход через границу происходит при $\eta=\sqrt{3} / 2$ и $\eta \sim(3 / \lambda)^{1 / 4} \rightarrow 0$ при $\lambda \rightarrow \infty$.

Переход к предельному режиму движения $\epsilon_{0}=\infty$, получаемый из уравнения (12), в котором положено $k=1 / 2$, определяется соотношением

$$
\lambda \eta^{4}-\eta^{2}+2=0
$$

откуда следует уравнение границы

$$
\lambda=\frac{\eta^{2}-2}{\eta^{4}}
$$

Здесь пересечение с осью $\lambda=0$ происходит в точке $\eta=\sqrt{2}$ и существует точка максимума $\lambda=1 / 8$, в которой $\eta=2$. За точкой максимума $\lambda \sim 1 / \eta^{2} \rightarrow 0$ при $\eta \rightarrow \infty$.

Линии уровня $\epsilon_{0}=$ const в пространстве $\lambda-\eta$ показаны на рисунке 7 вместе с границами перехода к различным режимам движения, которые определяются соотношениями $(17 b)$ и $(18 b)$. Горизонтальная пунктирная кривая соответствует случаю однородного сплошного диска.

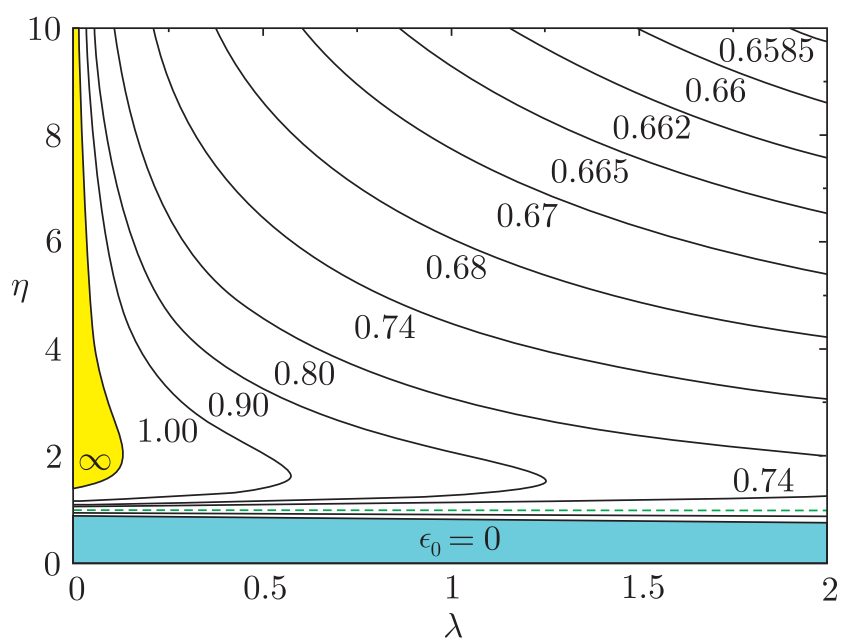

Рис. 7. Линии уровня $\epsilon_{0}$ в пространстве $\lambda-\eta$. Горизонтальная пунктирная линия $\eta=1$ соответствует случаю однородного диска. На рисунке также показаны границы перехода к режимам $\epsilon_{0}=0$ и $\epsilon_{0}=$ $=\infty$, определяемые уравнениями $(17 b)$ и $(18 b)$.

Пример эволюции линейной скорости и угловой скорости для $k=3.0>\sqrt{2 / 3}$ показан на рисунке 8. Здесь изменение $v^{*}$ снова представлено сплошной кривой, а изменение $\omega^{*}-$ пунктирной кривой. Так как $k>\sqrt{2 / 3}$, то в соответствии с результатами, изложенными в таблице 1, скольжение должно прекращаться раньше, чем вращение. Однако это не наблюдается в численном эксперименте, в котором мы обнаружили, что скольжение и вращение заканчиваются одновременно в момент $t_{f}^{*}=14.656$, обозначенный жирной точкой на рисунке 8. Однако в этом случае диск движется с очень малой скоростью в течение значительного интервала времени до того момента, как он окончательно остановится.

Эта особенность финального движения может быть объяснена, если исследовать динамические уравнения движения в окрестности значения $\epsilon=0$. Используя разложения в ряд 


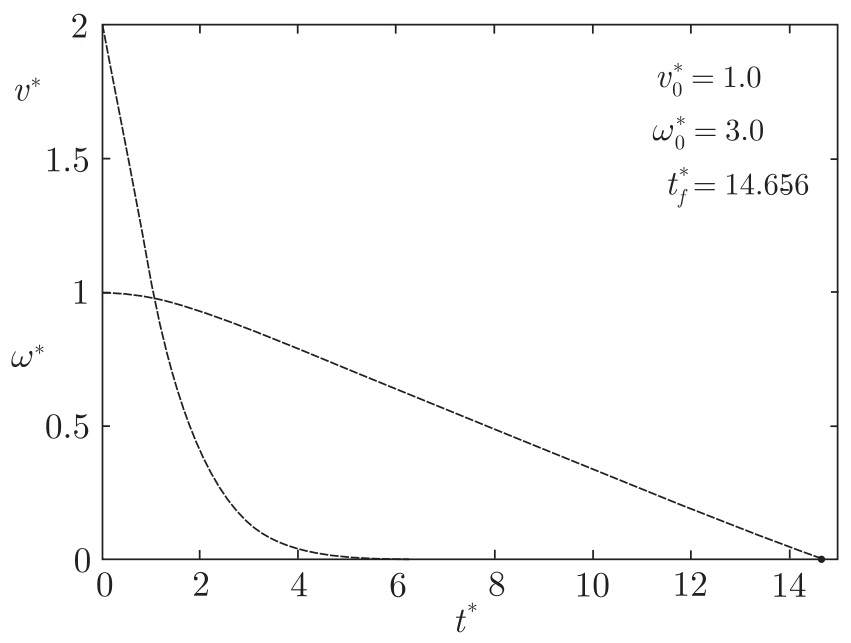

Рис. 8. График зависимости от безразмерного времени $t^{*}$ линейной $v^{*}$ (сплошная линия) и угловой $\omega^{*}$ (пунктирная линия) скоростей для составного диска с параметром $k=3$.

по $\epsilon$ для функций $K(\epsilon)$ и $E(\epsilon)$, приведенные в [14], мы находим, что соответствующие ряды для $\mathcal{F}(\epsilon)$ и $\mathcal{T}(\epsilon)$ имеют вид

$$
\left\{\begin{array}{l}
\mathcal{F}(\epsilon) \sim \epsilon-\frac{1}{8} \epsilon^{3}+\cdots \\
\mathcal{T}(\epsilon) \sim \frac{2}{3}-\frac{1}{2} \epsilon^{2}+\cdots
\end{array}(\epsilon \rightarrow 0) .\right.
$$

В этих разложениях сохраним только члены не выше первого порядка малости. Вспоминая, что $\epsilon=v^{*} / \omega^{*}$, и используя (12), динамические уравнения (13a) и (13b) вблизи значения $\epsilon=0$ приближенно запишем так:

$$
\begin{aligned}
\frac{d v^{*}}{d t^{*}} & =-\frac{v^{*}}{\omega^{*}}, \\
\frac{d \omega^{*}}{d t^{*}} & =-\frac{2}{3 k^{2}} .
\end{aligned}
$$

Явное решение этих уравнений легко объясняет предельное поведение системы:

$$
\begin{aligned}
& v^{*}=C\left(t_{f}^{*}-t^{*}\right)^{3 k^{2} / 2}, \\
& \omega^{*}=\frac{2}{3 k^{2}}\left(t_{f}^{*}-t^{*}\right),
\end{aligned}
$$

где $C$ - неопределенная постоянная и $t_{f}^{*}$ - момент остановки. Разделив $(21 a)$ на $(21 b)$ получим уравнение, определяющее изменение $\epsilon$ вблизи точки остановки:

$$
\epsilon=K\left(t_{f}^{*}-t^{*}\right)^{\left(3 k^{2}-2\right) / 2} \quad\left(t^{*} \rightarrow t_{f}^{*}\right)
$$

для некоторой постоянной $K$. Так как в $(21 c) \epsilon \rightarrow \epsilon_{0}=0$ только для $k>\sqrt{2 / 3}$, мы получаем подтверждение результата, приведенного в таблице 1 . Как это обычно бывает при такого типа исследовании, постоянная $C$ в $(21 a)$ может быть определена только приравниванием выражения $(21 a)$ и значения $v^{*}$ вблизи $t_{f}^{*}$, полученного из численного эксперимента. Хотя 
линейное и угловое движения исчезают одновременно, согласно уравнениям (20) угловое ускорение вблизи момента остановки постоянно, тогда как линейное ускорение пропорционально $\epsilon$ и в соответствии с $(21 c)$ мало в окрестности $t_{f}^{*}$. Более того, так как показатель степени в уравнении $(21 a)$ пропорционален $k^{2}$, эта окрестность может быть довольно большой, даже для значений $k$, лишь немного превосходящих значение $\sqrt{2 / 3}$, как показано на рисунке 8.

На рисунке 9 показана эволюция скорости и угловой скорости диска для $k=0.25<1 / 2$, соответствующего режиму $\epsilon_{0}=\infty$. На рисунке $9 \mathrm{a}$, на котором $v_{0}^{*}>\omega_{0}^{*}$, кривые не пересекаются до момента остановки $t_{f}^{*}=2.009$, тогда как на рисунке $9 \mathrm{~b}$ для $v_{0}^{*}<\omega_{0}^{*}$ кривые пересекаются до того момента, как происходит остановка при $t_{f}^{*}=1.137$. Здесь снова линейная $v^{*}$ и угловая $\omega^{*}$ скорости обращаются в нуль одновременно при $t^{*}=t_{f}^{*}$, и момент остановки обозначен на рисунке жирной точкой. Однако значения угловой скорости перед остановкой будут очень малы. Это поведение может быть объяснено исследованием динамических уравнений вблизи $\epsilon=\infty$, где $\mathcal{F}(\epsilon)$ и $\mathcal{T}(\epsilon)$ из $(3 a)$ и $(3 b)$ имеют следующее асимптотическое поведение:

$$
\left\{\begin{array}{l}
\mathcal{F}(\epsilon) \sim 1-\frac{1}{8} \frac{1}{\epsilon^{2}}+\cdots \\
\mathcal{T}(\epsilon) \sim \frac{1}{4} \frac{1}{\epsilon}+\cdots
\end{array}(\epsilon \rightarrow \infty) .\right.
$$

В этом случае уравнения движения могут быть приближенно записаны в виде

$$
\begin{gathered}
\frac{d v^{*}}{d t^{*}}=-1, \\
\frac{d \omega^{*}}{d t^{*}}=-\frac{1}{4 k^{2}} \frac{\omega^{*}}{v^{*}},
\end{gathered}
$$

откуда находится их решение

$$
\begin{gathered}
v^{*}=\left(t_{f}^{*}-t^{*}\right), \\
\omega^{*}=C\left(t_{f}^{*}-t^{*}\right)^{1 / 4 k^{2}},
\end{gathered}
$$

где снова неопределенная постоянная $C$ может быть получена только из сравнения этого решения с результатами численного эксперимента. Предельное поведение отношения скоростей $\epsilon$ определяется формулой

$$
\epsilon=K\left(t_{f}^{*}-t^{*}\right)^{\left(4 k^{2}-1\right) / 4 k^{2}} \quad\left(t^{*} \rightarrow t_{f}^{*}\right) .
$$

Здесь, в противоположность случаю $\epsilon=0$, угловая скорость $\omega^{*}$ может принимать очень малые значения перед тем, как обратится в нуль при $t^{*}=t_{f}^{*}$. Это находит подтверждение при анализе рисунка 10, на котором виден рост $\epsilon$ при приближении к моменту остановки. Начальные условия при этом были выбраны такими же, как для рисунка 9.

Полученные выше результаты относительно финального движения для режимов $\epsilon_{0}=0$ и $\epsilon_{0}=\infty$ показывают, что линейная и угловая скорости всегда обращаются в нуль одновременно, хотя одна из этих переменных может принимать малые значения на конечном промежутке времени перед тем, как диск остановится. 

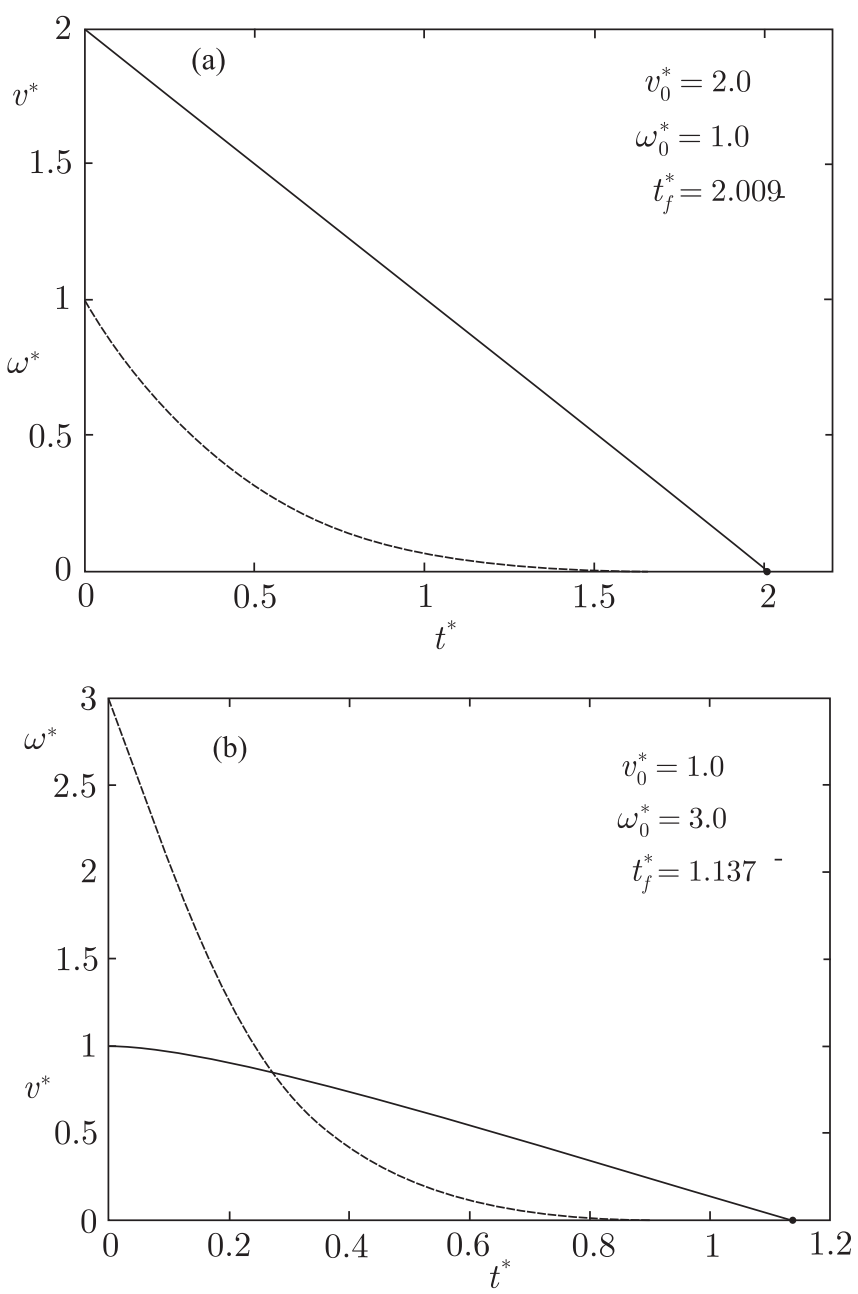

Рис. 9. График зависимости от безразмерного времени $t^{*}$ линейной $v^{*}$ (сплошная линия) и угловой $\omega^{*}$ (пунктирная линия) скоростей для составного диска с параметром $k=0.25$ при $v_{0}^{*}>\omega_{0}^{*}(\mathrm{a})$ и при $v_{0}^{*}<\omega_{0}^{*}(\mathrm{~b})$.

\section{5. Эксперимент}

Мы изучили финальное поведение некоторых составных дисков экспериментально. В нашем распоряжении имелся алюминиевый оптический стол размера $4 \times 10$ футов, изображенный на рисунке 11. Плексигласовый лист однородной толщины 0.25 дюйма и размером $2 \times 10$ футов был закреплен в центральной части стола при помощи самоклеющихся полосок толщиной не более 0.001 дюйма. Плексигласовый лист был обтянут плотно прилегающей необработанной нейлоновой тканью, которая была прикреплена к столу по периметру листа. Движение дисков записывалось на видеокамеру Olympus i-Speed с частотой 100 кадров в секунду. Для уменьшения эффекта параллакса камера записывала изображение, отражающееся в зеркале, расположенном под углом 45 градусов к столу. При таком расположении зеркала камера могла отобразить участок листа длиной 4 фута и шириной 1 фут. По границе участка, который фиксировался камерой, были положены черные и белые измерительные полоски с целью маркировки пикселов в направлении движения. Лампы с высокой интенсивностью света были установлены на пути движения диска и обеспечивали освещение, 


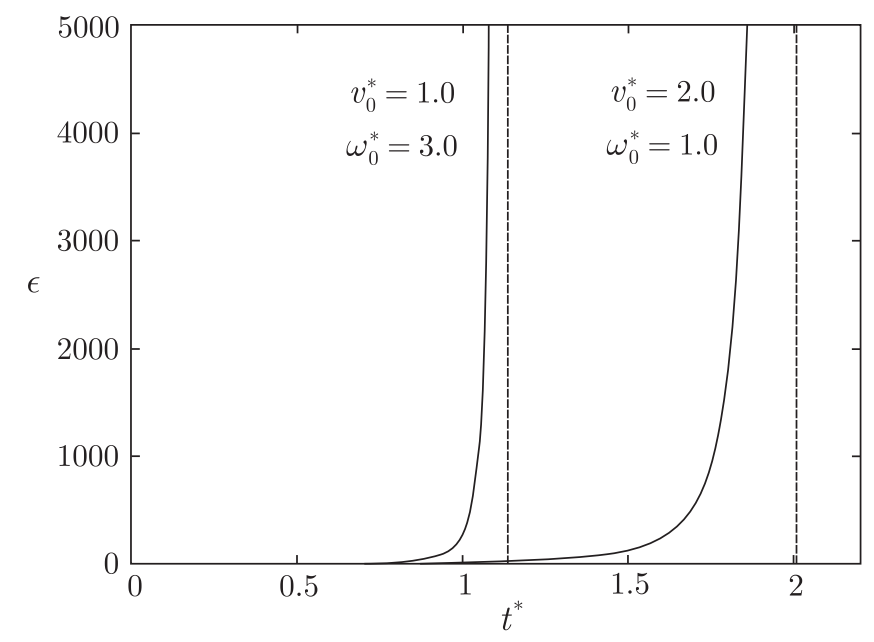

Рис. 10. Быстрый рост отношения скоростей $\epsilon$ при $k=0.25$ и тех же начальных условиях, что и на рисунке 9. Время до остановки равно $t_{f}^{*}=1.137$ для левой кривой и $t_{f}^{*}=2.009$ для правой кривой.

достаточное для того, чтобы на каждом отдельном кадре можно было точно определить положение диска.

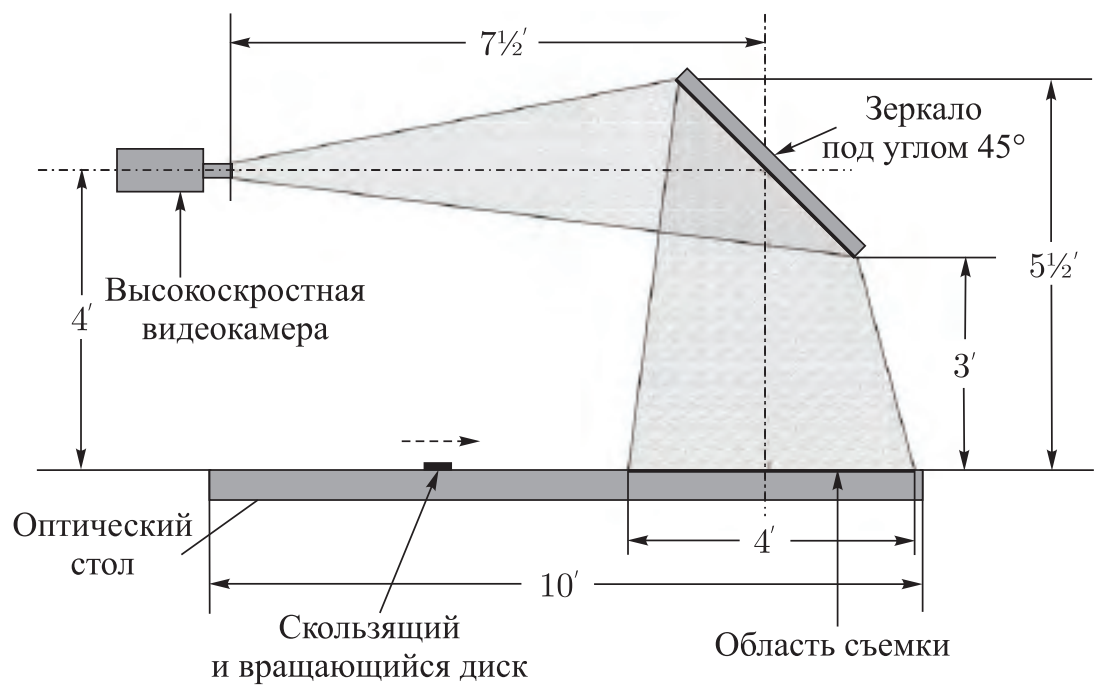

Рис. 11. Схема экспериментальной установки.

Так как простейшая теория, используемая нами для описания движения диска, предполагает равномерное распределение давления по площадке контакта, нам необходимо выработать некоторые критерии для применения этой теории. ${ }^{1}$ Линия действия нормальной силы реакции отстоит на расстояние $\bar{R}$ от вертикальной прямой, проходящей через центр

\footnotetext{
${ }^{1}$ Данное утверждение авторов ошибочно: учет ненулевой высоты дисков приводит к зависимости сил реакции не только от динамических параметров $\lambda$ и $\eta$, но и от кинематического параметра $\epsilon$. Подробнее см. статью Иванова А. П. в журнале «Прикладная математика и механика», 2009, т. 73, вып. 2, с. 189-203. - Прим. ред.
} 
масс, в направлении движения вследствие замедления диска, вызванного трением скольжения. Приложение законов Ньютона дает для этого расстояния формулу

$$
\bar{R}=\frac{\mu H_{2}}{2}\left(\frac{\eta^{2} \lambda^{2}+2 \lambda+1}{\eta^{2} \lambda+1}\right) .
$$

и распределение давлений по площадке контакта будет примерно равномерным, если $\bar{R} / R_{1}-$ малая величина.

Черными ромбиками в конфигурационном пространстве на рисунке 12 показаны значения параметра $\eta$, выбранные для примера в каждой из областей финальных движений, соответствующих прямой $\lambda=0.1$. Составные диски были подобраны так, что все они имели примерно одинаковое значение параметра $\bar{R} / R_{1} \approx 0.1$. Коэффициент трения первоначально оценивался как $\mu=0.5$. Отношение радиусов было выбрано следующее: $\eta=0.8$ в области $\epsilon_{0}=0, \eta=1.0$ (однородный диск), $\eta=1.25$ в области, где скольжение и вращение прекращаются одновременно, и $\eta=2.0$ в области $\epsilon_{0}=\infty$. Диски были изготовлены из ПВХ, и их контактные поверхности были гладко отшлифованы при помощи автоматического шлифовального камня, с последовательным использованием все более мелких сортов наждачной бумаги. Реальный коэффициент трения, полученный как среднее значение коэффициентов трения, измеренных для всех четырех дисков, составил $\mu=0.39 \pm 0.05$. Величины $R_{1}, H_{1}, \lambda$, $\eta$ и $k$ приведены в таблице 2 и упорядочены по возрастанию $\eta$; туда же включены величины $\bar{R} / R_{1}$ (вычисленные для измеренных значений $\mu$ ) и предсказываемые теорией величины для $\epsilon_{0}-$ неподвижной точки функции $f(\epsilon ; k)($ см. $(11))$.

Таблица 2. Геометрические характеристики составных дисков, использовавшихся в эксперименте (Примечание: диск 2 - однородный и сплошной)

\begin{tabular}{cccccccc}
\hline & $R_{1}$ (дюймы) & $H_{1}$ (дюймы) & $\lambda$ & $\eta$ & $k$ & $\bar{R} / R_{1}$ & $\epsilon_{0}$ \\
\hline Диск 1 & 1.15 & 0.040 & 0.10 & 0.50 & 1.401 & 0.082 & 0 \\
Диск 2 & 2.00 & 0.875 & 0.10 & 1.00 & 0.707 & 0.171 & 0.653 \\
Диск 3 & 2.00 & 0.075 & 0.10 & 1.25 & 0.587 & 0.079 & 0.970 \\
Диск 4 & 2.20 & 0.100 & 0.10 & 2.00 & 0.482 & 0.081 & $\infty$ \\
\hline
\end{tabular}

Кроме силы трения Кулона, мы наблюдали в опыте две дополнительные силы. Статическое электричество возникало от того, что ПВХ-диск двигался по нейлоновой ткани. Этот эффект был минимизирован за счет того, что перед каждым запуском диск помещался на алюминиевый стол.

Значительно более серьезная проблема была связана с силой адгезии (прилипания), возникающей при плотном взаимодействии поверхностей. Значительная вертикальная сила необходима, чтобы предотвратить «всасывание» диска в плоскость при его движении по хорошо обработанной нейлоновой ткани. Хотя среднее значение коэффициента трения $\mu$ для дисков, скользящих по хорошо обработанной нейлоновой ткани достаточно мало, влияние силы сцепления на предельное движение диска 4 (наиболее легкого из всех дисков, но обладающего наибольшей площадью контакта) было значительным. По этой причине мы выбрали более грубую нейлоновую ткань с коэффициентом трения $\mu=0.39-$ наибольшим из перечисленных выше, но на такой ткани адгезия проявляется существенно меньше, чем на хорошо обработанной ткани. 


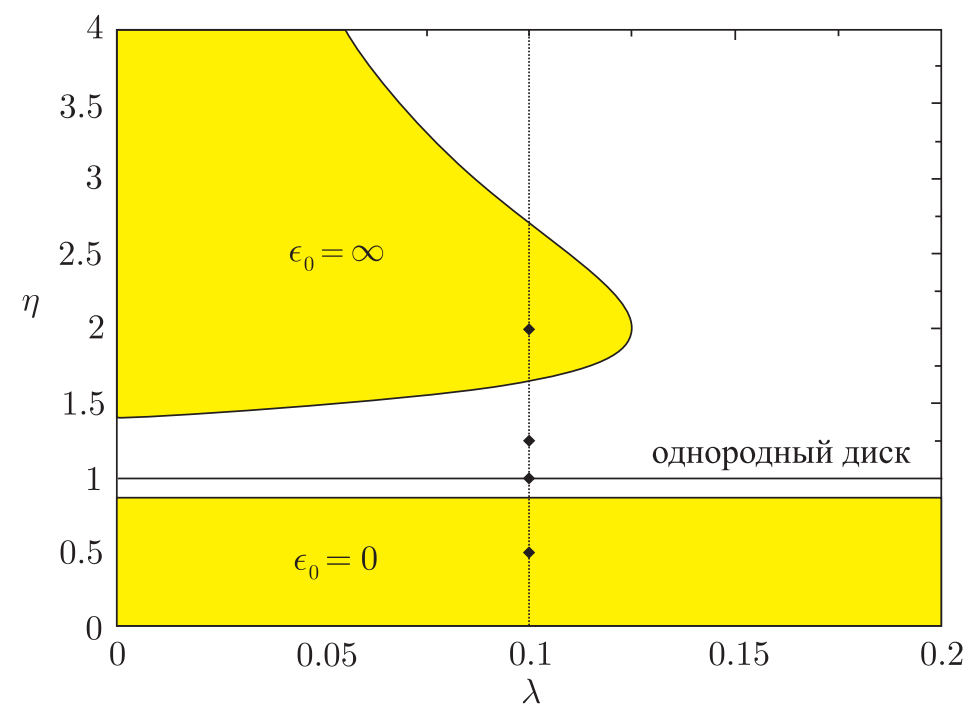

Рис. 12. Границы областей с различными режимами движения в пространстве $\lambda-\eta$ для составного диска. Черные ромбики соответствуют значениям $\eta$ при $\lambda=0.1$ для тех дисков, которые использовались в экспериментах.

Хотя сила сцепления должна изменять идеальное движение дисков, предсказанное теорией (особенно на завершающей стадии, где сцепление проявляется максимально сильно), прежде всего это влияние приводит к изменению величины силы трения и не оказывает существенного воздействия на качественное поведение диска, которое нас интересует.

Снимки предельного движения четырех дисков приведены на рисунках 13 и 14. Каждый коллаж сформирован из фотографий одних и тех же частей стола, рассматриваемых сверху, а диск движется справа налево. Промежутки времени между двумя последовательными снимками равны 0.7 с для рисунка 13a, 0.67 с для рисунка $13 \mathrm{~b}, 1$ с для рисунка 14a и 0.43 с для рисунка 14b. На рисунке 13 изображено финальное движение двух дисков, для которых значение $\epsilon_{0}$ конечно. Составной диск 3 на рисунке 13а останавливается через 0.23 с после кадра «и», и сплошной диск 2 на рисунке $13 \mathrm{~b}$ останавливается через 0.3 с после кадра «к». Рисунки 14a и 14b показывают финальное движение составных дисков для $\epsilon_{0}=0$ и $\epsilon_{0}=\infty$ соответственно. Диск 1 на рисунке 14а прекращает скольжение через 0.7 с после кадра «е» и прекращает вращение через 0.77 с после кадра «л». Диск 4 на рисунке $14 \mathrm{~b}$ прекращает вращение через 0.23 с после кадра «ж» и прекращает скольжение через 0.13 с после кадра «и». Эти экспериментальные результаты показывают, что в действительности скольжение и вращение диска прекращаются не одновременно, в отличие от предсказания математической теории.

На рисунке 15 представлены результаты некоторых количественных измерений финальных движений, сделанных для трех дисков. В каждом случае точками обозначены результаты экспериментальных данных, а линиями - их аппроксимация, выполненная по методу наименьших квадратов. На рисунке 15а представлены экспериментальные данные эволюции со временем отношения скоростей $\epsilon$ для сплошного диска 2. Хотя диск действительно останавливается вблизи теоретически предсказанной точки $\epsilon_{0}=0.653$, теоретический момент остановки $t_{f}^{*}$, вычисленный с использованием первого значения $\epsilon$, на рисунке $15 \mathrm{a}$ примерно на $10 \%$ больше, чем наблюдается в эксперименте. Данные на рисунке 15b описывают финальное движение дисков 1 и 4 . Эти эксперименты могут дать лишь качественное подтверждение имеющейся теории, поскольку последняя не учитывает ни влияние силы 


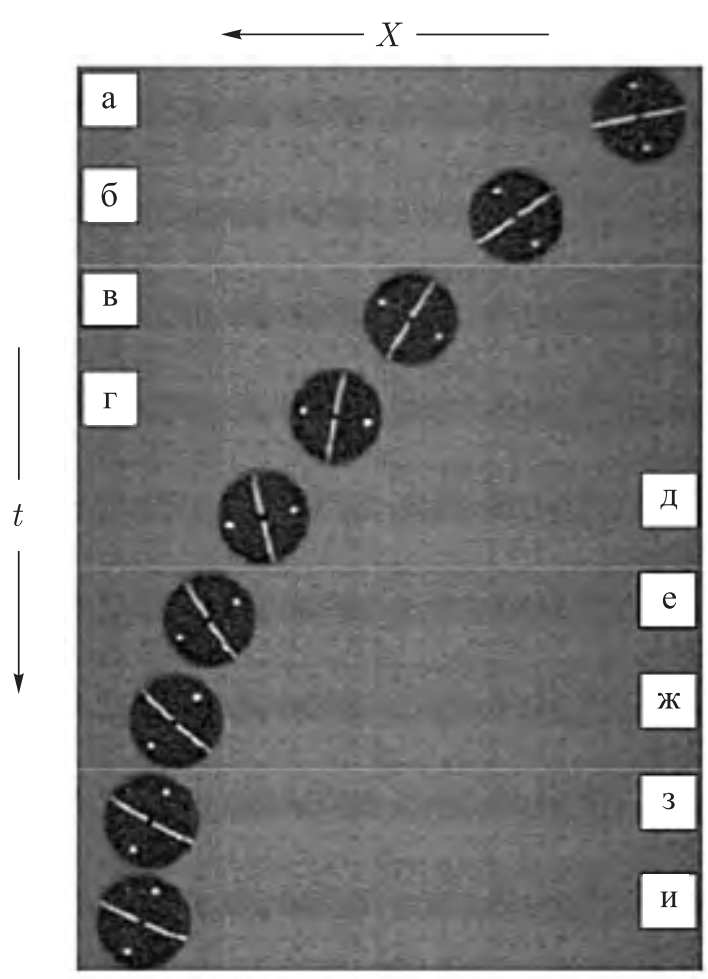

(a)

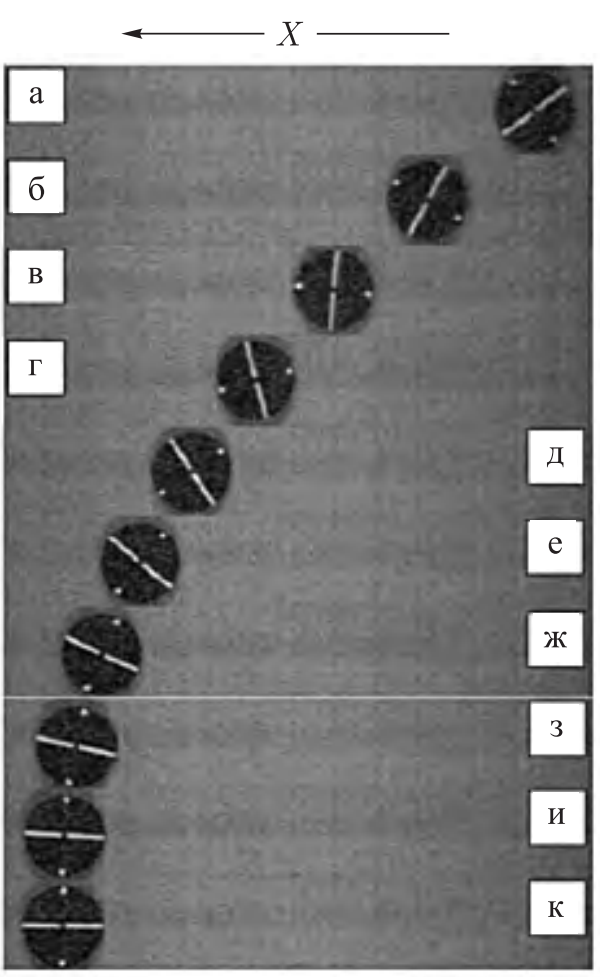

(b)

Рис. 13. Снимки, показывающие, что поступательное и вращательное движения прекращаются одновременно для (а) составного диска 3 с расстоянием по горизонтали 30 дюймов и интервалом между кадрами 0.70 с и для (b) сплошного диска 2 с горизонтальным расстоянием в 29 дюймов и интервалом между кадрами 0.67 с. Более подробно см. в тексте статьи.

сцепления, ни ненулевое значение величины $\bar{R}$. Действительно, теоретические вычисления, использующие первую из величин $\epsilon$ в качестве начальных данных, на рисунке 15b дают величину времени до остановки на 10-15\% большую, чем наблюдается в эксперименте.

Наконец, замечания заслуживает следующий наблюдаемый в наших экспериментах эффект: при $\epsilon_{0}=0$ скольжение прекращается явно раньше вращения, а при $\epsilon_{0}=\infty$ вращение прекращается явно раньше скольжения. Например, анализ промежуточных точек, не изображенных на рисунке $15 \mathrm{~b}$, показывает, что скорость изменения $\epsilon_{1}$ конечна около значения $t_{f}^{*}$, которое приблизительно равно $t_{1}^{*}=12$. То же самое выполняется при рассмотрении графика функции $\epsilon_{4}^{-1}$ в зависимости от $t^{*}$, построенного по результатам, приведенным на рисунке $15 \mathrm{~b}$, где не показано несколько точек, взятых для $\epsilon_{4}>48$. Итак, в противоположность всем теоретическим рассуждениям о скользящих дисках и кольцах, в которых скольжение и вращение прекращаются одновременно (возможно, с отношением скоростей, стремящихся к 0 или к $\infty$ ), мы наблюдаем чистое предельное движение на конечном интервале времени: в одном случае это поступательное движение, а в другом чистое вращение.

В работах $[2,3]$ было показано, что чистое вращение или поступательное движение существуют при условии, что предельная поверхность имеет грани (плоские участки). Для изотропного трения дисков, кольцевых дисков и колец, рассмотренных выше, предельная поверхность не имеет граней. Грани могут возникать по двум причинам, которые мы сейчас и обсудим. 


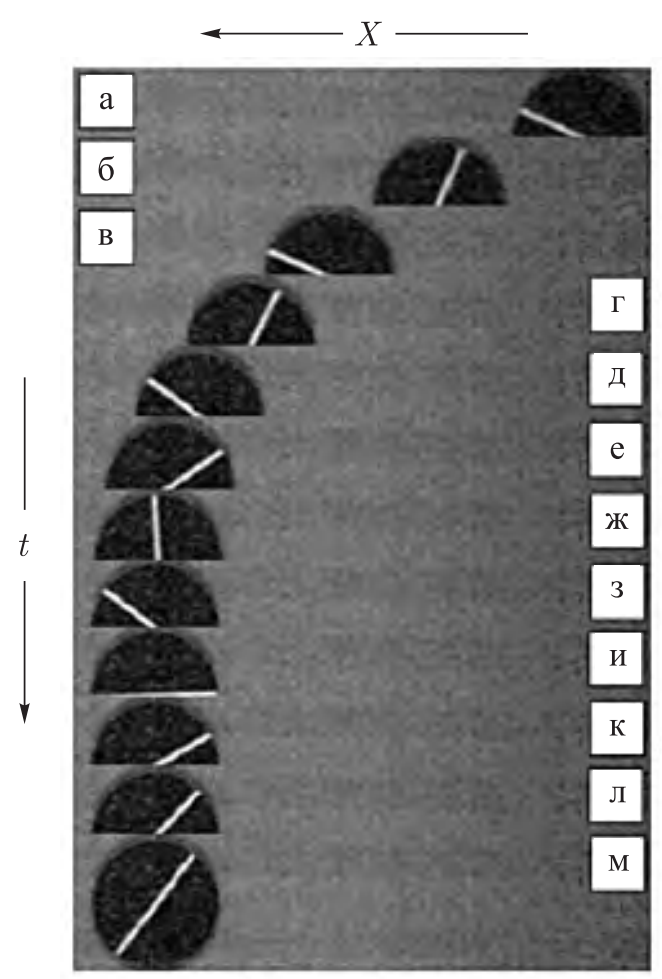

(a)

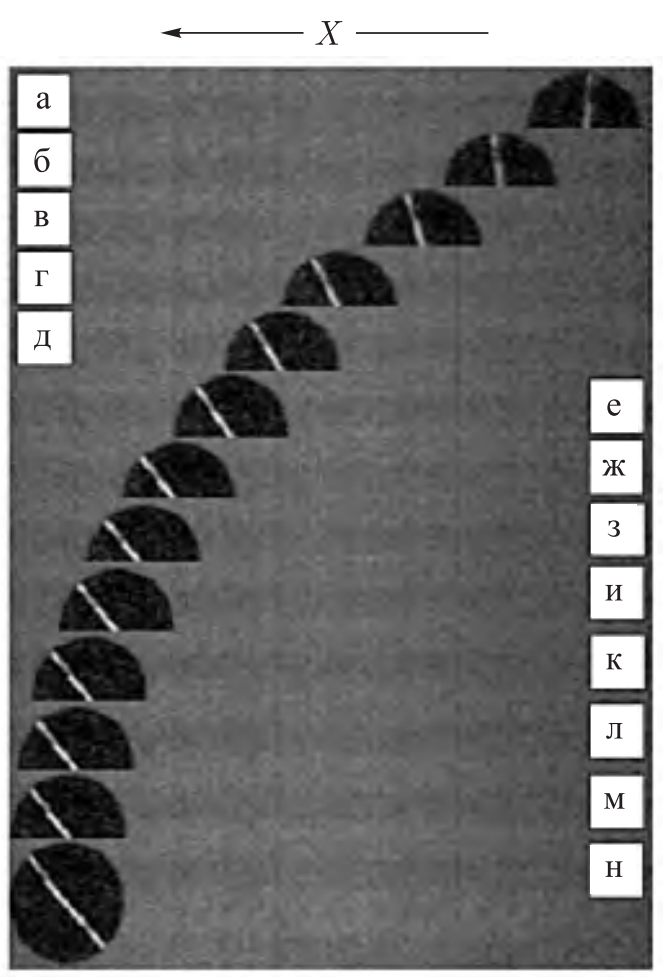

(b)

Рис. 14. Снимки, показывающие финальное поведение: а) диска 1, для которого скольжение прекращается раньше вращения (ракурс по горизонтали 21 дюйм, интервал между кадрами 1 с), и b) диска 4, для которого вращение прекращается раньше скольжения (ракурс по горизонтали 27 дюймов, интервал между кадрами 0.43 с). Более подробно см. в тексте статьи.

1. Сосредоточенная опора. Если нормальная нагрузка конечна на бесконечно малом участке контакта, то предельная поверхность всего движущегося тела имеет грань. Так, например, у перевернутого фрисби (игрушки «летающая тарелка») внешняя поверхность зачастую имеет невысокий штырек в центре поверхности. В результате движение фрисби по плоскости таково, что вращение продолжается в течение конечного времени после прекращения скольжения (Гойял, частное сообщение). Однако мы исключаем эту возможность как объяснение полученного нами экспериментального результата: поверхности имевшихся у нас дисков были хорошо обработаны и не имели признаков сосредоточенной силы или износа в центральной точке.

2. Анизотропное трение. Даже если трение не является сосредоточенным, оно может приводить к наличию граней у предельной поверхности. Например, равномерное распределение для прямых коньков или равномерное распределение для закругленных коньков приводят к наличию граней на предельной поверхности. Одним из объяснений существования чистых финальных движений может служить то, что в процессе движения трение может менять свой характер с изотропного на анизотропный, что порождает грани у предельной поверхности в окрестности финального движения. Это формальное описание согласуется с интуитивным представлением о возникновении на трущихся поверхностях желобков в направлении скольжения. Анизотропия обусловлена тем, что трение при движении вдоль желобков меньше, чем при движении в поперечном направлении. Однако изучение развития анизотропного трения для тестовых поверхностей не было целью данной работы. 


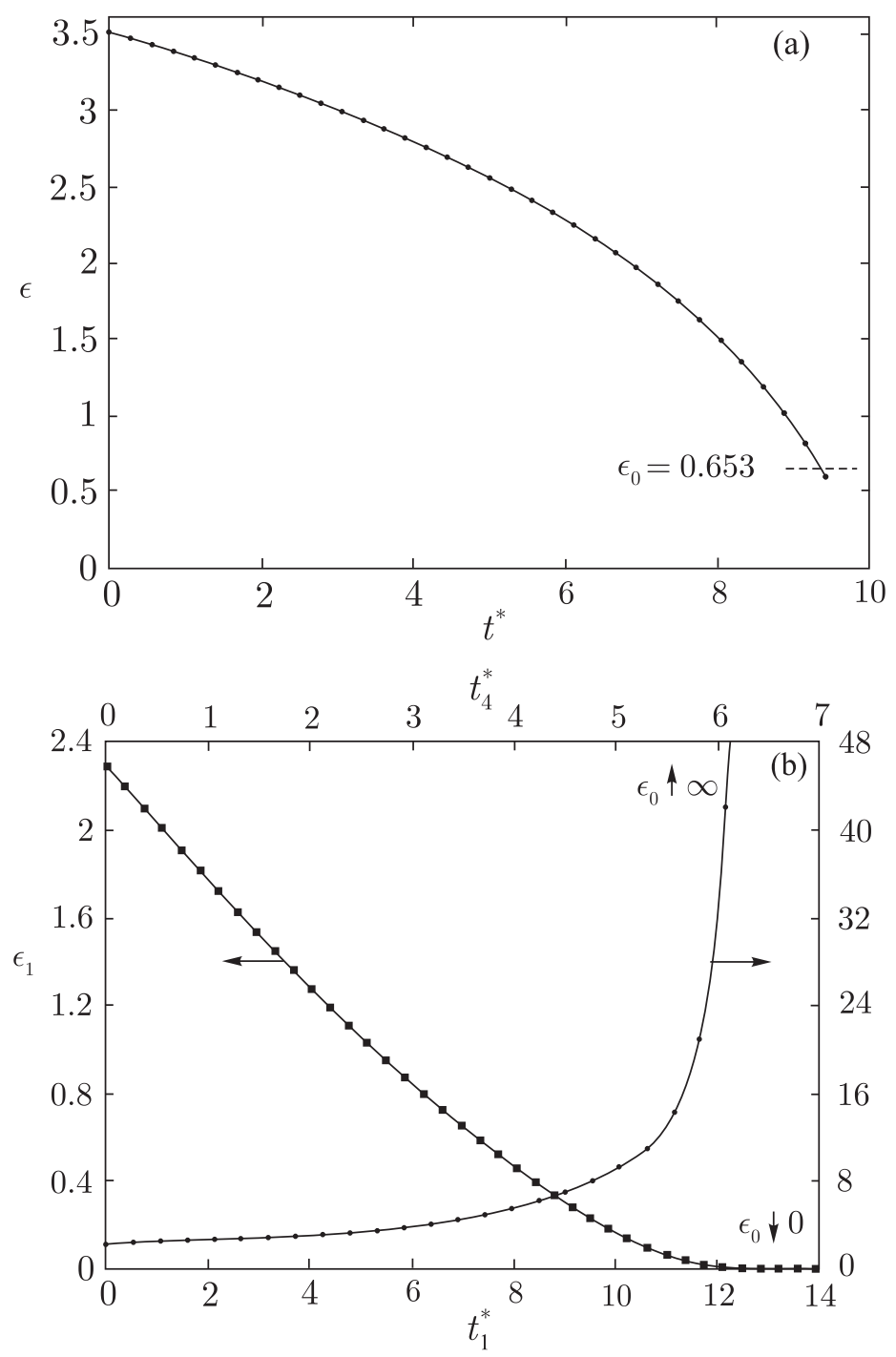

Рис. 15. Измерения, показывающие зависимость от времени отношения скоростей $\epsilon$ для экспериментов с сплошным диском 2 (а) и составными дисками 1 и 4 (b).

\section{6. Выводы}

Теоретическое исследование, проведенное на основе аналитического подхода из статьи Фаркаша с соавторами [8], имело целью изучить финальное движение диска, геометрия которого отлична от геометрии сплошного диска. Кольцевые диски, характеризуемые параметром $\eta$ - отношением внутреннего радиуса к внешнему, всегда прекращают вращение и поступательное движение одновременно, и отношение предельных скоростей $\epsilon_{0}=v / \omega R_{2}$ меняется от значения 0.653, соответствующего случаю сплошного диска, до значения 1.0 для кольца. Таким образом, для тонкого кольца предельное поведение удовлетворяет условию $v=R \omega$, полученному ранее в работах А. Ю.Ишлинского с соавторами [4], Военли и Эриксена [5], а также Гойяла с соавторами [2, 3].

Наша цель заключалась в том, чтобы подобрать диск с геометрией, отличной от геометрии обычного диска, чтобы для него имело место финальное поведение, отличное от фи- 
нального поведения, описанного в [8]. Эта цель была достигнута при помощи составного диска, который характеризовался двумя параметрами - отношением радиусов $\eta$ и отношением толщин $\lambda$. Для такого составного диска можно проанализировать характер предельного движения в зависимости лишь от одного параметра $k$ - безразмерного радиуса инерции, т. е. радиуса инерции $\sqrt{I / m}$, поделенного на радиус $R_{1}$ диска, находящегося в контакте с плоскостью. Наш анализ показал, что отношение $\epsilon_{0}$ будет конечным числом для всех дисков, для которых $1 / 2 \leqslant k \leqslant \sqrt{2 / 3}$; таким образом, для этих дисков скольжение и вращение прекращаются одновременно. Неожиданным результатом, полученным при помощи асимптотических методов, оказался результат, что при $k>\sqrt{2 / 3}$ в режиме $\epsilon_{0}=0$ и при $k<1 / 2$ в режиме $\epsilon_{0}=\infty$ диски также прекращают скольжение и вращение одновременно. Такая однопараметрическая зависимость финального движения осесимметрических дисков с распределением масс, зависящим от радиуса, была ранее установлена Гойялом с соавторами [3] на основе теории предельных поверхностей, изложенной в $[2,6]$. Наш асимптотический анализ показывает, однако, что скольжение в режиме $\epsilon_{0}=0$ и вращение в режиме $\epsilon_{0}=\infty$ будут алгебраически малыми, хотя и ненулевыми.

В дополнение к нашим новым результатам по динамике кольцевого диска мы укажем, как наша работа развивает предшествующие исследования. Прежде всего, мы в явном виде указали формулы асимптотического поведения для финального движения дисков с переменным распределением масс в режимах $\epsilon_{0}=0$ и $\epsilon_{0}=\infty$. В режиме $\epsilon_{0}=0$ угловая скорость стремится к нулю линейно со временем, тогда как линейная скорость стремится к нулю по степенному закону с показателем $3 k^{2} / 2$ для $k>\sqrt{2 / 3}$. В режиме $\epsilon_{0}=\infty$ скорость скольжения убывает линейно со временем, тогда как угловая скорость стремится к нулю по степенному закону с показателем $1 / 4 k^{2}$ для $k<1 / 2$. Во-вторых, мы аналитически в пространстве параметров $\eta-\lambda$ указали границы перехода от одного режима к другому для составного диска и вычислили предельное отношение скоростей в случае, когда $\epsilon_{0}$ является ненулевой константой. Тем самым мы представили отношение скоростей $\epsilon_{0}$ как функцию безразмерного радиуса инерции $k$ для произвольных осесимметричных тел, скользящих и вращающихся на плоскости с изотропным сухим трением. В-третьих, нами были представлены экспериментальные данные об эволюции движения дисков со временем для каждого из предельных режимов в двух различных форматах. В первом формате были представлены фотографии различных положений диска при его поступательно-вращательном движении. Во втором формате сделанные на основе этих фотографий измерения использовались для того, чтобы проанализировать изменение со временем отношения скоростей $\epsilon$.

Совершенно новый результат был получен при экспериментальном рассмотрении движения дисков. Оказывается, при действительном движении диска его скольжение (вращение) полностью прекращается, тогда как движение, дополнительное к данному (т. е. вращение (скольжение)), продолжается еще в течение конечного промежутка времени. На наш взгляд, этот эффект можно объяснить лишь изменением характера трения при скольжении поверхностей (анизотропией).

Тот факт, что одно движение полностью прекращается до того, как прекратится другое, означает, что только два диска необходимы для демонстрации всех трех режимов предельного движения дисков. Можно воспользоваться дисками 2 и 4, параметры которых приведены в таблице 1. Сплошной диск 2 будет прекращать свое скольжение и вращение, как показано на рисунке 15 в в условиях нашего эксперимента. Диск 4 при $\epsilon_{0}=\infty$ в какой-то момент прекращает вращение и скользит до остановки. Если мы теперь перевернем этот диск и положим его на плоскость другой, малой стороной, то величины $\eta$ и $\lambda$ для такого случая будут обратны значениям, приведенным в таблице 1. Таким образом, геометрические 
параметры для перевернутого диска 4 будут $\eta=0.5$ и $\lambda=10$. Величина $\eta_{b}$, ограничивающая режим $\epsilon_{0}=0$ и получающаяся из уравнения $(17 b)$, будет $\eta_{b}(\lambda=10)=0.619$. Так как $\eta<\eta_{b}$, этот перевернутый диск должен вести себя так, как предсказано экспериментом для режима $\epsilon_{0}=0$. Мы выполнили эксперимент и убедились, что перевернутый диск 4 сначала прекращает скользить, а потом в течение конечного промежутка времени вращается до остановки.

В заключение хотелось бы напомнить читателю, что наш анализ осесимметричных кольцевых и составных дисков был ограничен малым отношением $\bar{R} / R_{1}$, т.е. распределение давления по площадке контакта предполагалось близким к однородному. В работе Фаркаша с соавторами [8] было отмечено, что представляет интерес задача изучения предельных движений тел, для которых отношение $F_{n} / A$ нельзя вынести из-под интегралов в формулах (1) и (2), так как при движении распределение давлений будет неоднородным.

\section{7. Благодарности}

Первый автор выражает свою признательность профессору Бруно Келлеру за гостеприимство и поддержку в течение летнего семестра 2004 года, когда автор работал приглашенным профессором на кафедре физики зданий Высшей технической школы г. Цюриха (Швейцария). Данное исследование было задумано как раз в то время. Авторы благодарят также Юджина Мадьяри и Гарри Томаса за полезные дискуссии и за результаты, которые описываются уравнением (4) данной работы. Особую благодарность за обсуждения на всех этапах работы авторы выражают Энди Руине и Сурешу Гойялу. Мы также благодарим за полезные советы Анджея Хержинского с физического факультета Бостонского колледжа. Все эксперименты, описанные в работе, были проведены при финансовой поддержке факультета прикладной механики Университета штата Колорадо, г. Боулдер.

\section{8. Приложение. Задача о движении тонкого кольца}

Рассмотрим одновременное скольжение и вращение тонкого кольца радиуса $R$ на горизонтальной плоскости. Предположим, что сверху к кольцу приклеена не имеющая массы доска. Масса системы равномерно распределена сверху доски по окружности произвольного радиуса $k^{\prime}$, поэтому величина $k=k^{\prime} / R$ представляет собой нормализованный радиус инерции данной системы, тогда как давление на опорную плоскость распределено по кольцу радиуса $R$.

Можно легко показать, что неподвижные точки данной системы также определяются уравнением (11), хотя, в противоположность уравнениям $(3 a)$ и $(3 b)$, функции $\mathcal{F}(\epsilon)$ и $\mathcal{T}(\epsilon)$ определяются теперь формулами

$$
\mathcal{F}(\epsilon)=\left\{\begin{array}{l}
\frac{4}{\pi \epsilon}\left[\left(\epsilon^{2}-1\right) K\left(\epsilon^{2}\right)+E\left(\epsilon^{2}\right)\right], \quad \epsilon \leqslant 1, \\
\frac{4}{\pi} E\left(\epsilon^{-2}\right), \quad \epsilon \geqslant 1,
\end{array}\right.
$$

и

$$
\mathcal{T}(\epsilon)=\left\{\begin{array}{l}
\frac{4}{\pi} E\left(\epsilon^{2}\right), \quad \epsilon \leqslant 1, \\
\frac{4 \epsilon}{\pi}\left[\left(\epsilon^{-2}-1\right) K\left(\epsilon^{-2}\right)+E\left(\epsilon^{-2}\right)\right], \quad \epsilon \geqslant 1 .
\end{array}\right.
$$

Эти результаты были получены из интегралов (1) и (2) после учета того, что движущееся тело является тонким кольцом единичного радиуса. 


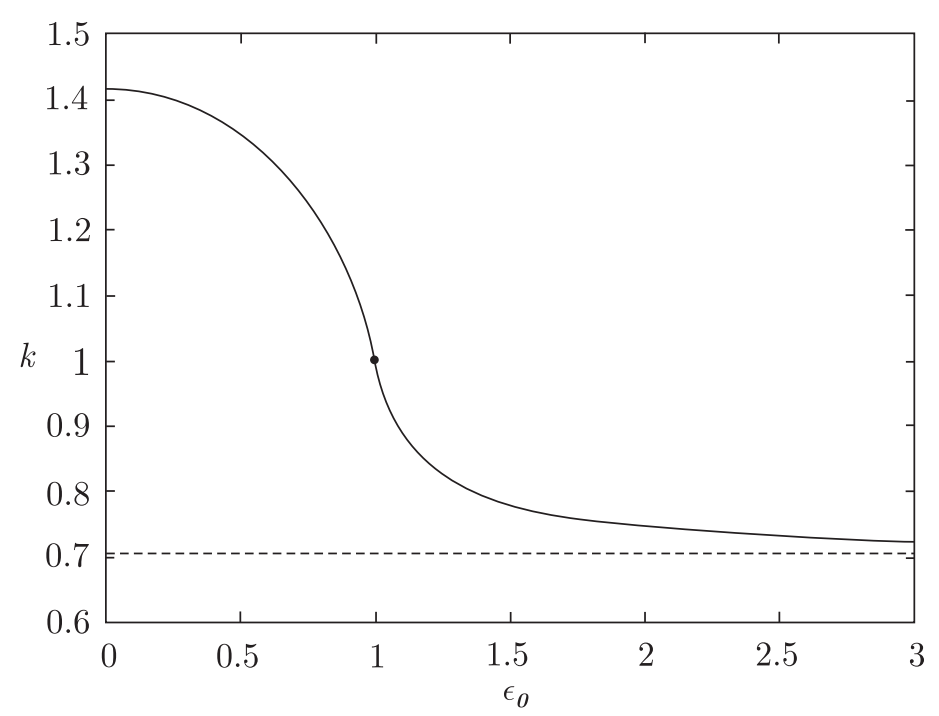

Рис. 16. Зависимость параметра $k$ от финального отношения скоростей $\epsilon_{0}$ для тонкого кольца с переменным распределением масс. Точка, для которой $k=1.0$ и $\epsilon_{0}=1.0$, представляет собой предел $\eta \rightarrow 1$ кольцевого диска, рассмотренного в части 3 статьи.

Используя разложения в ряд для $K(\epsilon)$ и $E(\epsilon)$ из [14], мы находим первые два члена разложения в ряд функций $\mathcal{F}(\epsilon), \mathcal{T}(\epsilon), \mathcal{F}^{\prime}(\epsilon), \mathcal{T}^{\prime}(\epsilon)$ для малых $\epsilon$ в виде

$$
\left\{\begin{array}{ll}
\mathcal{F}(\epsilon) \sim \epsilon-\frac{3}{8} \epsilon^{3}, & \mathcal{F}^{\prime}(\epsilon) \sim 1-\frac{9}{8} \epsilon^{2} \\
\mathcal{T}(\epsilon) \sim 2-\frac{1}{2} \epsilon^{2}, & \mathcal{T}^{\prime}(\epsilon) \sim-\epsilon-\frac{3}{8} \epsilon^{3}
\end{array}(\epsilon \rightarrow 0) .\right.
$$

Используя тот факт, что функция $f(\epsilon ; k)$ касается оси $\epsilon$ в точке, соответствующей границе между различными режимами предельного движения (см. рис. 5), дифференцированием уравнения (11) по $\epsilon$ и приравниванием полученного результата к нулю находим величину $k$, соответствующую значению на границе между двумя различными движениями:

$$
k=\sqrt{\frac{\mathcal{T}(\epsilon)^{2}}{\mathcal{T}(\epsilon) \mathcal{F}^{\prime}(\epsilon)-\mathcal{F}(\epsilon) \mathcal{T}^{\prime}(\epsilon)}},
$$

где штрих означает производную по $\epsilon$. Подставляя соотношения $(A .3)$ в $(A .4)$, находим, что переход к режиму $\epsilon_{0}=0$ происходит при $k=\sqrt{2}$.

Аналогичная процедура производится для получения параметра $k$ при переходе к режиму $\epsilon_{0}=\infty$. Сначала запишем разложения в ряд основных функций:

$$
\left\{\begin{array}{ll}
\mathcal{F}(\epsilon) \sim 2-\frac{1}{2 \epsilon^{2}}, & \mathcal{F}^{\prime}(\epsilon) \sim \frac{1}{\epsilon^{3}}-\frac{3}{8 \epsilon^{5}} \\
\mathcal{T}(\epsilon) \sim \frac{1}{\epsilon}+\frac{1}{8 \epsilon^{3}}, & \mathcal{T}^{\prime}(\epsilon) \sim-\frac{1}{\epsilon^{2}}-\frac{3}{8 \epsilon^{4}}
\end{array}(\epsilon \rightarrow \infty) .\right.
$$

Подставляя разложения $(A .5)$ в $(A .4)$ и переходя к пределу при $\epsilon \rightarrow \infty$, получаем $k=$ $=1 / \sqrt{2}$. В промежутке изменения $k$, равном $1 / \sqrt{2} \leqslant k \leqslant \sqrt{2}$, финальное движение кольца 
характеризуется конечными ненулевыми значениями отношения предельных скоростей $\epsilon_{0}$. Изменение $k$ в зависимости от $\epsilon_{0}$, вычисленное подстановкой соотношений $(A .1)$ и $(A .2)$ в уравнение (13), показано на рисунке 16.

Таким образом, мы описали предельные режимы тонкого кольца с переменным распределением масс при помощи аналитических методов. Наши результаты согласуются с результатами, полученными ранее в работе Гойяла с соавторами [3] и кратко перечисленными нами во введении к этой статье. Кроме того, напомним, что $R$ из работы [3] соответствует величине $1 / k$.

\section{Список литературы}

[1] Жуковский Н. Е. Условие равновесия твердого тела, опирающегося на неподвижную плоскость некоторой площадкой и могущего перемещаться вдоль этой плоскости с трением // Собр. соч.: Т. 1. М.-Л.: ГТТИ, 1948. С. 339-354.

[2] Goyal S., Ruina A., Papadopoulos J. Planar sliding with dry friction: P. 1. Limit surface and moment function // Wear, 1991, vol. 143, pp. 307-330.

[3] Goyal S., Ruina A., Papadopoulos J. Planar sliding with dry friction: P. 2. Dynamics of motion // Wear, 1991, vol. 143, pp. 331-352.

[4] Ишлинский А.Ю., Соколов Б.Н., Черноусько Ф.Л. О движении плоских тел при наличии сухого трения // Изв. РАН. МТТ, 1981, № 4, с. 17-28.

[5] Voyenli K., Eriksen E. On the motion of an ice hockey puck // Amer. J. Phys., 1985, vol. 53, pp. $1149-1153$.

[6] Mason M. T. Mechanics of robotic manipulation. Cambridge, MA: MIT Press, 2001. $272 \mathrm{pp}$.

[7] Goyal S. Planar sliding of a rigid body with dry friction: Limit surfaces and dynamics of motion: Ph. D. Thesis. Cornell University, Ithaca, 1989.

[8] Фаркаш 3., Бартельс Г., Унгер Т., Вольф Д. О силе трения при поступательном и вращательном движении плоского тела // Нелинейная динамика, 2011, т. 7, № 1, с. 139-146.

[9] Halsey T. C. Mechanics: Friction in a spin // Nature, 2003, vol. 424, pp. 1005-1006.

[10] Kadau D., Bartels G., Brendel L., Wolf D. E. Pore stabilization in cohesive granular systems // Phase Transitions, 2003, vol. 76, nos. 4-5, pp. 315-331.

[11] Knight J. B., Fandrich G. C., Lau C. N., Jaeger H. M., Nagel S. R. Density relaxation in a vibrated granular material // Phys. Rev. E, 1995, vol. 51 pp. 3957-3963.

[12] Nicodemi M., Coniglio A., Herrmann H. J. Frustration and slow dynamics of granular packings // Phys. Rev. E, 1997, vol. 55, pp. 3962-3969.

[13] Weidman P. D., Malhotra C. P. Regimes of terminal motion of sliding spinning disks // Phys. Rev. Lett., 2005, vol. 95, no. 26, 264303, 4 pp.

[14] Abramowitz M., Stegun I. Handbook of mathematical functions. Washington, DC: US Government Printing Office, 1972. $1046 \mathrm{pp}$.

[15] Wolfram S. Mathematica: A system for doing mathematics by computer. 2nd ed. Boston: AddisonWesley, 1991. $961 \mathrm{pp}$.

\section{On the terminal motion of sliding spinning disks with uniform Coulomb friction}

Patrick D. Weidman ${ }^{1}$, Chetan P. Malhotra ${ }^{2}$

${ }^{1}$ Department of Mechanical Engineering, University of Colorado, Boulder, CO 80309-0427, USA

${ }^{2}$ Tata Research Development and Design Centre, 54/B Hadapsar Industrial Estate, Pune 411013, India

${ }^{1}$ weidman@colorado. edu

We review previous investigations concerning the terminal motion of disks sliding and spinning with uniform dry friction across a horizontal plane. Previous analyses show that a thin circular 
ring or uniform circular disk of radius $R$ always stops sliding and spinning at the same instant. Moreover, under arbitrary nonzero initial values of translational speed $v$ and angular rotation rate $\omega$, the terminal value of the speed ratio $\epsilon_{0}=v / R \omega$ is always 1.0 for the ring and 0.653 for the uniform disk. In the current study we show that an annular disk of radius ratio $\eta=R_{1} / R_{2}$ stops sliding and spinning at the same time, but with a terminal speed ratio dependent on $\eta$. For a twotier disk with lower tier of thickness $H_{1}$ and radius $R_{1}$ and upper tier of thickness $H_{2}$ and radius $R_{2}$, the motion depends on both $\eta$ and the thickness ratio $\lambda=H_{1} / H_{2}$. While translation and rotation stop simultaneously, their terminal ratio $\epsilon_{0}$ either vanishes when $k>\sqrt{2 / 3}$, is a nonzero constant when $1 / 2<k<\sqrt{2 / 3}$, or diverges when $k<1 / 2$, where $\mathrm{k}$ is the normalized radius of gyration. These three regimes are in agreement with those found by Goyal et al. [S. Goyal, A. Ruina, J. Papadopoulos, Wear 143 (1991) 331] for generic axisymmetric bodies with varying radii of gyration using geometric methods. New experiments with PVC disks sliding on a nylon fabric stretched over a plexiglass plate only partially corroborate the three different types of terminal motions, suggesting more complexity in the description of friction.

Citation: Rus. J. Nonlin. Dyn., 2011, vol. 7, no. 2, pp. 339-365 (Russian)

Originally published in: Phys. D, 2007, vol. 233, no. 1, 248302. (C) Elsevier 